\title{
Identification of Creb3/4 as an essential negative regulator of adipogenesis
}

\author{
T-H Kim ${ }^{1}$, S-H Jo ${ }^{1,2}$, H Choi $^{1}$, J-M Park ${ }^{1}$, M-Y Kim ${ }^{1}$, H Nojima ${ }^{3}$, J-W Kim ${ }^{1}$ and Y-H Ahn ${ }^{*, 1,2}$
}

Understanding the molecular networks that regulate adipogenesis is crucial for combating obesity. However, the identity and molecular actions of negative regulators that regulate the early development of adipocytes remain poorly understood. In this study, we investigated the role of CREB3L4, a member of the CREB3-like family, in the regulation of adiposity. Constitutive overexpression of CREB3L4 resulted in the inhibition of adipocyte differentiation, whereas knockdown of Creb3/4 expression caused differentiation of preadipocytes into mature adipocytes, bypassing the mitotic clonal expansion step. In 3T3-L1 preadipocytes, Creb314 knockdown resulted in increased expression of peroxisome proliferator-activated receptor $\gamma$ (PPAR 2 ) and CCAAT/ enhancer binding protein (C/EBP $\alpha$ ), either by increasing the protein stability of C/EBP $\beta$ or by decreasing the expression of GATA3, a negative regulator of PPAR $\gamma 2$ expression. Consequently, increased PPAR $\gamma 2$ and C/EBP $\alpha$ levels induced adipocyte differentiation, even in the presence of minimal hormonal inducer. Thus, it can be speculated that CREB3L4 has a role as gatekeeper, inhibiting adipogenesis in 3T3-L1 preadipocytes. Moreover, adipocytes of Creb314-knockout mice showed hyperplasia caused by increased adipogenesis, and exhibited improved glucose tolerance and insulin sensitivity, as compared with littermate wild-type mice. These results raise the possibility that Creb3/4 could be a useful therapeutic target in the fight against obesity and metabolic syndrome. Cell Death and Disease (2014) 5, e1527; doi:10.1038/cddis.2014.490; published online 20 November 2014

Obesity is a worldwide epidemic in both developed and developing countries. Due to excess food intake, adipose tissue enlarges, via either hyperplasia or hypertrophy. ${ }^{1}$ Increased adipose tissue is associated closely with the development of insulin resistance and is the principal risk factor in the development of type 2 diabetes mellitus. ${ }^{2}$ Thus, studying the biological networks and transcription factors controlling adipogenesis, including adipocyte differentiation, may facilitate greater understanding of the pathophysiology of obesity and type 2 diabetes.

Adipogenesis is a multi-step process requiring a cascade of transcription factors that regulate the expression of genes involved in lipogenesis, $\beta$-oxidation, and fatty acid uptake. ${ }^{3}$ Members of the CCAAT/enhancer binding family, such as $\mathrm{C} /$ $\mathrm{EBP} a,-\beta$, and $-\delta$, have key roles in adipogenesis. ${ }^{4,5} \mathrm{C} / \mathrm{EBP} \beta$ and $\mathrm{C} / \mathrm{EBP} \delta$ directly upregulate the expression of C/EBP $a$ and peroxisome proliferator-activated receptor $\gamma$ (PPAR $\gamma) .{ }^{6}$ Upon expression, PPARy and C/EBPa cooperate to express the full adipogenic program, including induction of additional transcription factors and suppression of growth-associated genes. $^{7}$

Recently, endoplasmic reticulum (ER) membrane-bound bZIP domain-containing transcription factors, such as cyclic AMP-responsive element-binding protein 3(CREB3)/Luman/ LZIP, CREB3-like 1 (CREB3L1)/OASIS, BBF2H7/CREB3L2, CREBH/CREB3L3, and CREB4/CREB3L4, were identified. ${ }^{8}$
These proteins share a region of high sequence homology with activating transcription factor 6 (ATF6), having a transmembrane (TM), a transcription-activation, and a bZIP domain. CREB3L4 (also called AlbZIP, Tisp40, or ATCE1) is expressed in human prostate tissue, ${ }^{9}$ and $\mathrm{Creb} 3 / 4$ disruption resulted in abnormal epididymal sperm nuclei, ER stress, and activation of caspase 12, leading to apoptosis of meiotic/ postmeiotic germ cells. ${ }^{10}$

Mouse Creb3/4 generates CREB3L4 $a$ and CREB3L4 $\beta$, which differ in their 55-residue $\mathrm{N}$-terminal extension. Deletion of the TM domain of CREB3L4 results in retention of the protein in the nucleus. ${ }^{11}$ CREB3L $4 \beta$, but not CREB3L4a, activates transcription of ER degradation-enhancing $a$-mannosidase-like protein (Edem), which enhances unfolded protein degradation, presumably by acting on the unfolded protein response element (UPRE). ${ }^{11}$ The role of CREB3L4 in ER stress is beginning to be understood, while its role in adipocyte differentiation and obesity is unknown.

In this study, we demonstrate a novel role of CREB3L4 in adipogenesis. We demonstrate that CREB3L4 acts as a negative regulator of adipogenesis through dual mechanisms. Silencing of Creb3/4 potently increases adipocyte differentiation even in the presence of minimal hormonal inducer bypassing the mitotic clonal expansion (MCE) step, enhancing the PPARY2 expression through regulating protein stability of $\mathrm{C} / \mathrm{EBP} \beta$ and GATA3 expression in vitro. Mice with deleted

\footnotetext{
${ }^{1}$ Department of Biochemistry and Molecular Biology, Yonsei University College of Medicine, Seoul 120-752, Republic of Korea; ${ }^{2}$ Brain Korea 21 PLUS Project for Medical Sciences, Yonsei University College of Medicine, Seoul 120-752, Republic of Korea and ${ }^{3}$ Department of Molecular Genetics, Research Institute for Microbial Diseases, Osaka University, Osaka, Japan

*Corresponding author: Y-H Ahn, Department of Biochemistry and Molecular Biology, Yonsei University College of Medicine, 50-1 Yonsei-ro, Seodaemun-gu, Seoul 120-752, Republic of Korea. Tel: +82 22228 0835; Fax: +82 2312 5041; E-mail: yha111@yuhs.ac

Abbreviations: CREB3L4, cyclic AMP-responsive element-binding protein 3 (CREB3)-like 4; PPAR, peroxisome proliferator-activated receptor; MEF, mouse embryonic fibroblast; C/EBP, CCAAT/enhancer binding protein; MCE, mitotic clonal expansion

Received 15.8.14; revised 29.9.14; accepted 09.10.14; Edited by G Melino
} 
Creb3/4 showed improved metabolic parameters due to the increase in numbers of fat cells, caused by increased adipogenesis. These results provide a biological framework for discovery of potential target genes, such as Creb3/4, in obesity treatment.

\section{Results}

CREB3L4 is regulated during adipocyte differentiation. To investigate whether the Creb-like family is involved in energy metabolism, we first examined the expression of the corresponding genes in various metabolic tissues of mice. Creb3/3 is most abundantly expressed in the liver, ${ }^{12}$ but white adipose tissue (WAT) expressed markedly more Creb3/4 as compared with other tissues suggesting that WAT could be one of the target tissue of CREB3L4 action (Supplementary Figure S1). The expression of Creb3/4 in WAT led us to explore the role of CREB3L4 in adipocyte differentiation and the development of adiposity. Therefore, we wondered whether CREB3L4 is a regulatory component of the differentiation program, and whether its expression could be regulated during differentiation. During 3T3-L1 preadipocyte differentiation, Creb3/4 expression decreased from day 0 (D0) and recovered after D2, as shown by real-time PCR (Figure 1a) and western blotting (Figure 1b). Fractionation of adipose tissue into mature adipocytes (AdipoF) and the stromal-vascular fraction (SVF) revealed that Creb3/4 was more abundant in the SVF, which contains adipocyte precursors (Aps), than in AdipoF (Figure 1c). To determine which components of the differentiation cocktail were responsible for decreased Creb3/4 expression, 3T3-L1 cells were stimulated for 1 day with insulin (I), dexamethasone (D), or isobutyl methylxanthine (M), alone, or in combination (DMI). Protein level of CREB3L4 was decreased the most by DMI treatment, followed by treatment with isobutyl methylxanthine, and with dexamethasone (Figure 1d). These results suggested that CREB3L4 may have a critical role in adipocyte differentiation.

CREB3L4 acts as a negative regulator of adipogenesis. We next determined whether CREB3L4 affects adipogenesis in vitro. To investigate the function of CREB3L4, we transfected the nuclear form of Creb3/4 (Creb3/4-N) ${ }^{13}$ into 3T3-L1 cells; these cells showed reduced oil-red $\mathrm{O}$ staining (Figure 2a). Constitutive expression of Creb3/4-N in 3T3-L1 preadipocytes resulted in a decrease in $\mathrm{C} / \mathrm{EBP} \beta$ from D0, with a concomitant decrease in C/EBPa and PPARy2 expression (Figure 2b; Supplementary Figure S2). Overexpression of Creb3/4 decreased mRNA levels of Cebpa and Pparg2, but that of Cebpb did not change in the early stages (Figure 2c; discussed below), suggesting that CREB3L4 has a negative role during DMl-induced differentiation of preadipocytes into adipocyte.

Suppression of Creb3/4 expression resulted in accelerated adipocyte differentiation by upregulating PPARy2 expression in 3T3-L1 preadipocytes. If CREB3L4 has a negative role in adipogenesis in preadipocytes, then inhibition of Creb3/4 expression should result in accelerated a

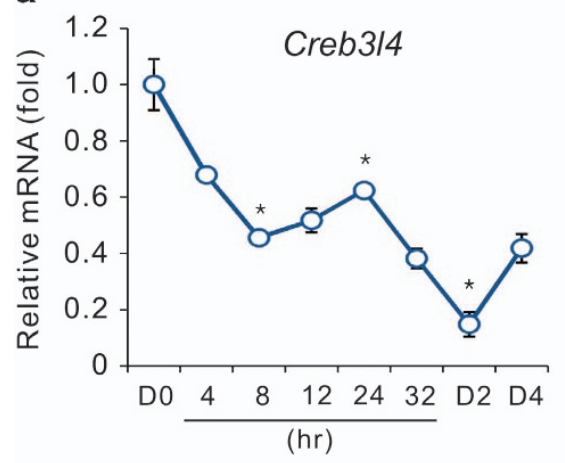

C

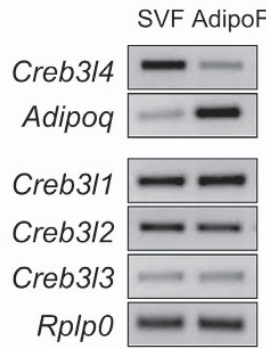

b

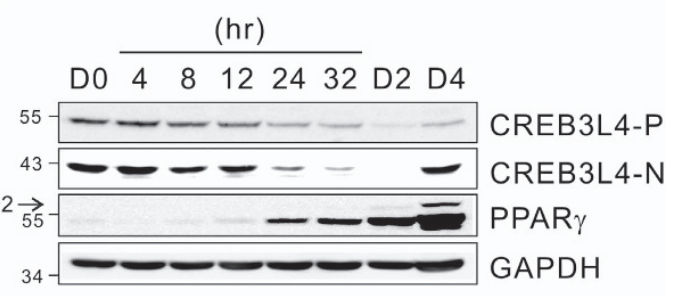

d

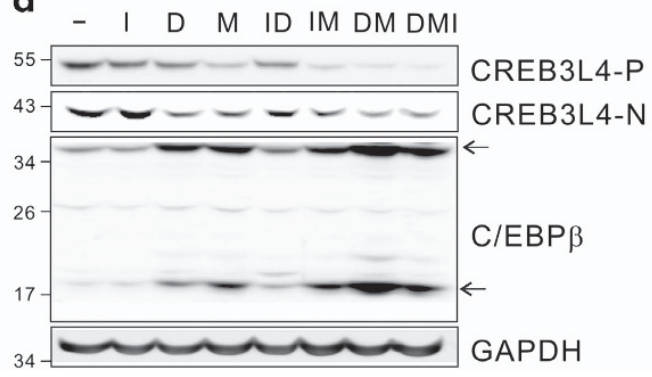

Figure 1 Regulation and function of CREB3L4 during adipocyte differentiation. (a) Real-time PCR analysis of Creb3/4 mRNA levels in 3T3-L1 cells treated with differentiation cocktail (DMI: $0.5 \mathrm{mM}$ 3-isobutyl-1-methylzanthine [IBMX; M], $1 \mu \mathrm{M}$ dexamethasone [D], and $1 \mu \mathrm{g} / \mathrm{ml}$ of insulin [l]) $(n=3)$. Error bars represent mean \pm S.E.M., ${ }^{*} P<0.05$. (b) Immunoblot of CREB3L4 during differentiation of 3T3-L1 preadipocytes, treated with DMI; CREB3L4-P, precursor form of CREB3L4; CREB3L4-N, nuclear form of CREB3L4. (c) Semi-quantitative RT-PCR analysis of the expression of the Creb3l family in the SVF and the AdipoF. SVF, stromal vascular fraction; AdipoF, adipose fat fraction. (d) Immunoblot of CREB3L4 and C/EBP $\beta$ in 3T3-L1 cells treated for 1 day with individual components of the differentiation cocktail 
a

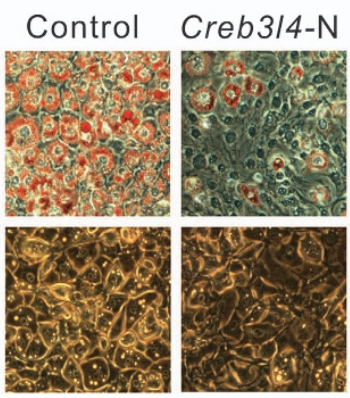

b
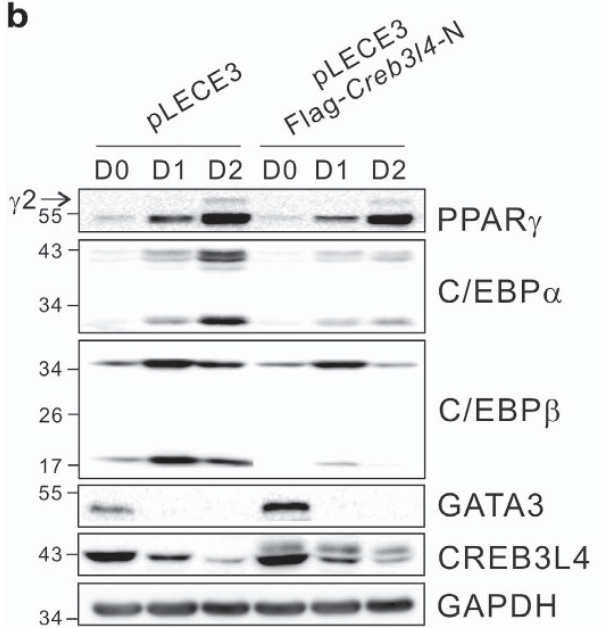

C
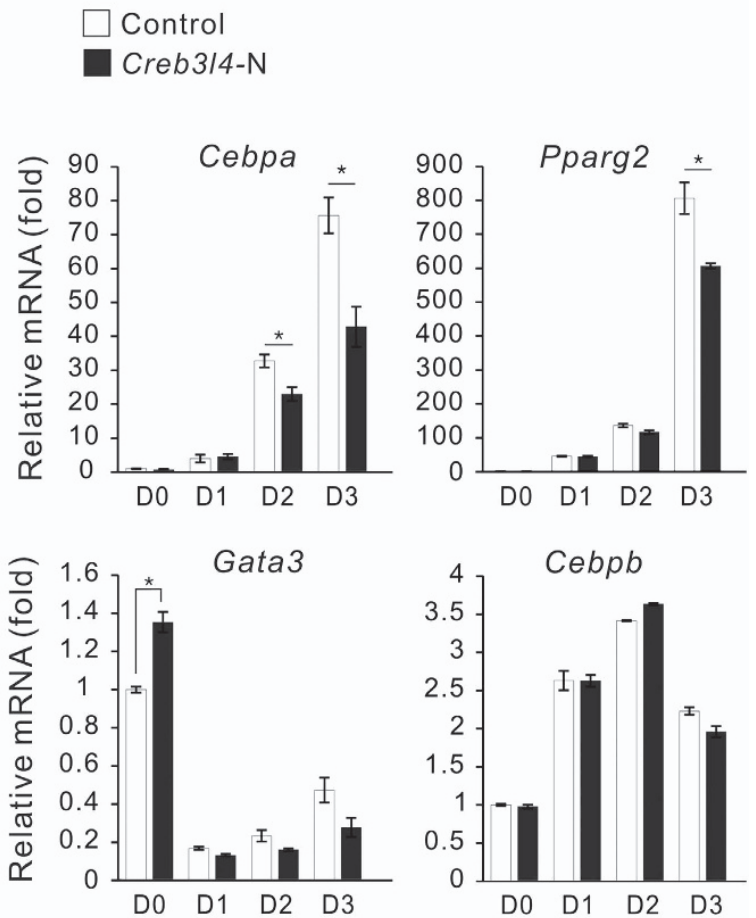

Figure 2 CREB3L4 inhibits the adipocyte differentiation. (a) 3T3-L1 cells were transfected with Creb3/4 (N) by electroporation, after which differentiation was induced by differentiation cocktail (DMl: $0.5 \mathrm{mM}$ 3-isobutyl-1-methylzanthine [IBMX; M], $1 \mu \mathrm{M}$ dexamethasone [D], and $1 \mu \mathrm{g} / \mathrm{ml}$ of insulin [I]). Oil-red O staining of 3T3-L1 cells expressing Creb3/4 (N) at day 8 (D8), and microscopy (x20) was performed. (b) 3T3-L1 cells were transduced with lentiviral Flag-Cre3/4 (N) and harvested at the indicated times. Expression of critical adipogenic factors, as determined by immunoblot. (c) The mRNA levels of Cebpa, Pparg2, Gata3, and Cebpb were measured by real-time PCR during 3T3-L1 differentiation. Their expression was normalized to that of the Rplpo (36B4) control. Values are expressed as mean \pm S.E.M., $n=3$ per group, ${ }^{*} P<0.05$

differentiation into adipocytes. To test this hypothesis, we examined the adipocyte differentiation capacity of cells under the influence of a minimal hormonal inducer, which suppressed differentiation in the scramble group (Figure 3a; Supplementary Figure S3a). To this end, 3T3-L1 cells that had been transfected with scramble small-interfering RNA (siRNA) or Creb3/4-siRNA (siCreb3/4) were mock treated, or treated with dexamethasone or insulin in Dulbecco's modified Eagle's medium (DMEM) containing fetal bovine serum (10\% FBSDMEM). The scramble group did not differentiate into adipocytes in the presence of the minimal inducer of differentiation, with mock, insulin, or dexamethasone treatment, whereas in the absence of the inducer, the siCreb3/4 group exposed to mock treatment differentiated partially (Supplementary Figure S3b). Furthermore, modified differentiation methods were sufficient to induce adipocyte differentiation in the siCreb3/4 group (Figure 3b; Supplementary Figure S3b). siCreb3/4-transfected cells that had been cultured in the presence of insulin showed an increase in protein levels of adipogenic transcription factor (Figure 3c), and PPARy2 and C/ EBPa levels were already elevated on D0. The mRNA level of Pparg2, Cebpa, and lipogenic genes for adipocyte differentiation, Ixra and Pepck, were also induced in siCreb3/4transfected 3T3-L1 cells (Figure 3d).
To further confirm the role of CREB3L4 in adipogenesis, we established a stable cell line expressing Creb3/4-shRNA (shCreb3/4); these cells showed increased oil-red O staining when cultured in the presence of insulin (Figure 3e). In addition, shCreb3/4 cells showed increased expression of PPARy2 and C/EBPa at D0 (Figures $3 f$ and g). These data indicated that suppression of Creb3/4 could enhance the capacity of preadipocytes to differentiate into mature adipocytes, and therefore CREB3L4 seemed to be a critical negative regulator, determining preadipocyte-adipocyte transition in 3T3-L1 cells.

Knockdown of Creb3/4 results in bypass of the MCE step. Since MCE of growth-arrested preadipocytes appears to be necessary for optimal differentiation, ${ }^{14}$ we wondered whether increased differentiation in siCreb3/4 group with insulin treatment underwent the MCE stage or not. To evaluate whether the siCreb3/4 group re-entered the cell cycle due to insulin treatment, we performed a Bromodeoxyuridine (BrdU) incorporation assay. DMI treatment of the scramble group resulted in active incorporation of BrdU, whereas treatment with insulin alone or with siCreb3/4 decreased BrdU incorporation as well as the number of cells (Figures $4 \mathrm{a}$ and b). Although the combination of siCreb3/4 
a

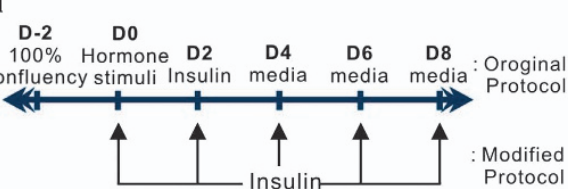

b
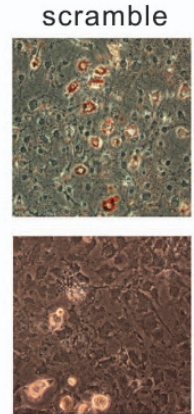

siCreb3/4
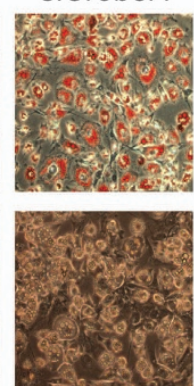

C

$\frac{\mathrm{D} 0}{+-\frac{\mathrm{D} 1}{+-}} \frac{\mathrm{D} 2}{+-\overline{\mathrm{D}} 3} \frac{\mathrm{D} 4}{+-}$ scramble -+-++-+-+ siCreb3/4

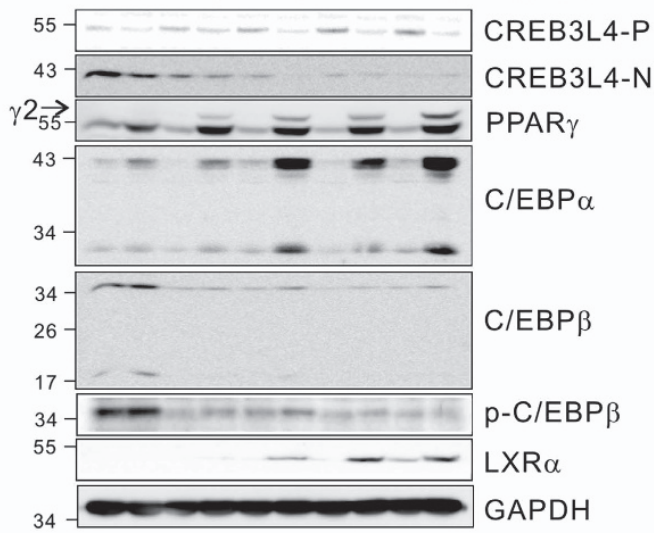

d
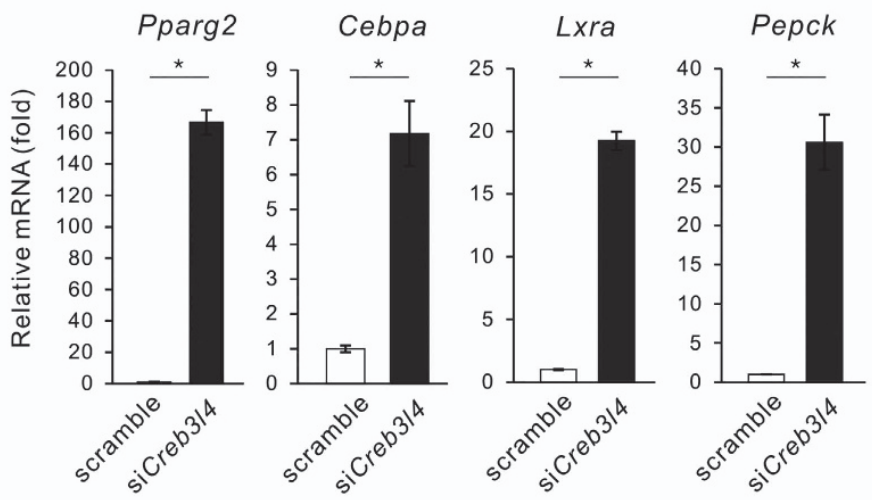

Creb3/4

e
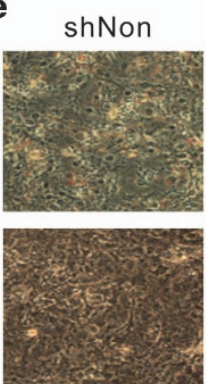

shCreb3/4

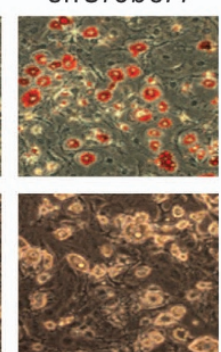

f

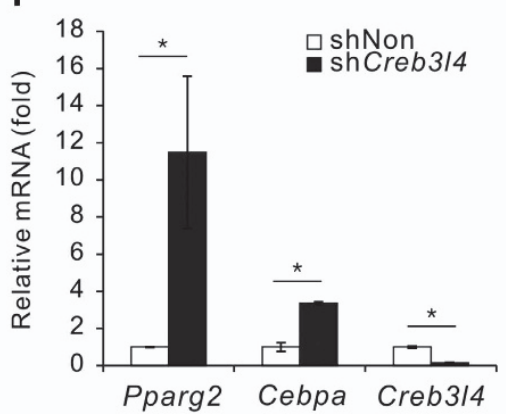

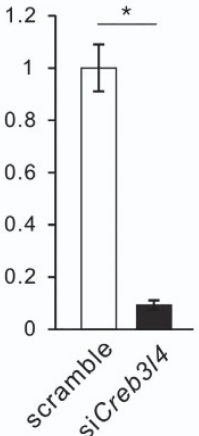

g

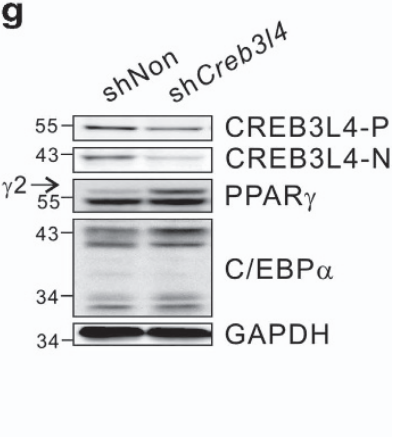

Figure 3 Effect of Creb3/4 ablation on adipocyte differentiation. (a) Modified protocol for inducing adipocyte differentiation. Induction of adipogenesis using the modified protocol in siCreb3/4-expressing 3T3-L1 cells treated with insulin ( $1 \mu \mathrm{g} / \mathrm{ml}$ )-containing media (10\% FBS-DMEM) every 2 days. (b) After 8 days, the cells were stained with oil-red 0 , and microscopy (x20) was performed. Microscopic views representative of three independent experiments are shown. (c) Immunoblot of adipogenic transcription factors at the indicated time points after induction of 3T3-L1 differentiation by insulin. (d) The mRNA levels related to lipogenesis, Ixra and Pepck, and adipogenesis, Pparg2 and Cebpa, were determined by real-time PCR at D6. (e) Oil-red O staining of stable 3T3-L1 cells expressing shCreb3/4 at D8, and microscopy (x20) was performed. The 3T3-L1 cells were transduced with lentiviral shCreb3/4 and treated with $10 \%$ FBS-DMEM media containing insulin ( $1 \mu \mathrm{g} / \mathrm{ml})$ every 2 days. Real-time PCR (f) and immunoblot (g) of adipogenic gene expression in 3T3-L1 cells expressing shCreb3/4 at D0. Their expression was normalized to that of the Rplp0 (36B4) control. GAPDH was used as a loading control. Data are representative of three independent experiments. Values are expressed as mean \pm S.E.M., $n=3$ per group, ${ }^{*} P<0.05$

and insulin treatment was not able to increase cell proliferation and cell number, adipocyte differentiation still occurred (Figures 3b, 4a, and b). Treatment of DMl to induce differentiation of 3T3-L1 preadipocytes undergo two sequential rounds of mitosis over the next 2 days. ${ }^{15}$ As illustrated in Figure 4c, when the MCE step was skipped, scramble group did not induce adipogenesis. However, adipogenesis in Creb3/4 knockdown cells was intact and adipogenic transcription factor, Pparg2 and Cebpa, was increased compared with scramble group (Figures $4 \mathrm{c}$ and d). Surprisingly, knockdown of Creb3/4 in 3T3-L1 cells resulted in bypassing MCE during adipocyte differentiation. 
a

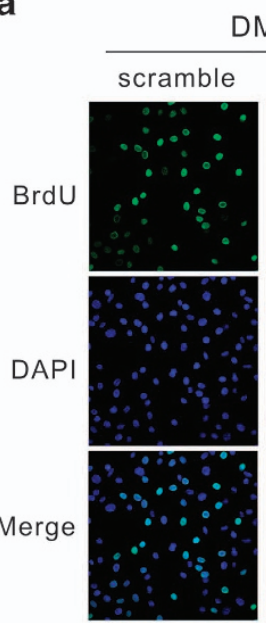

DMI

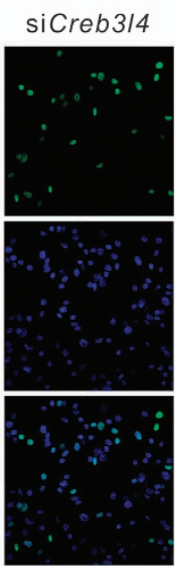

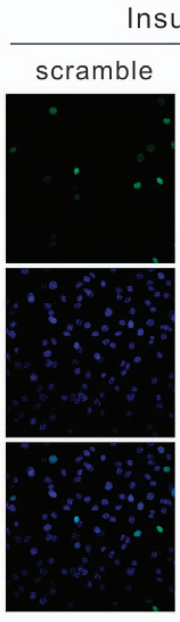

b

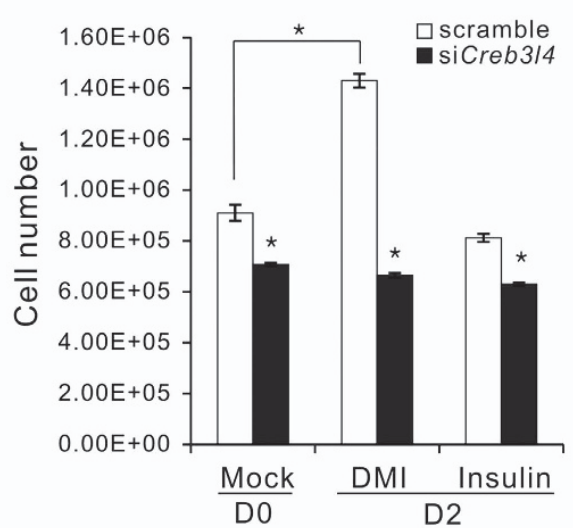

C

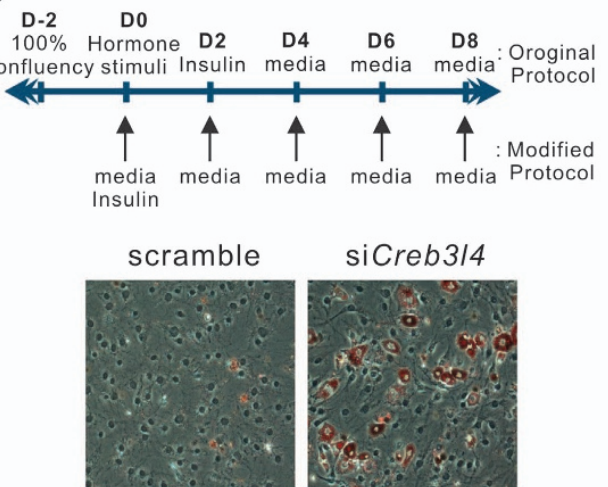

d
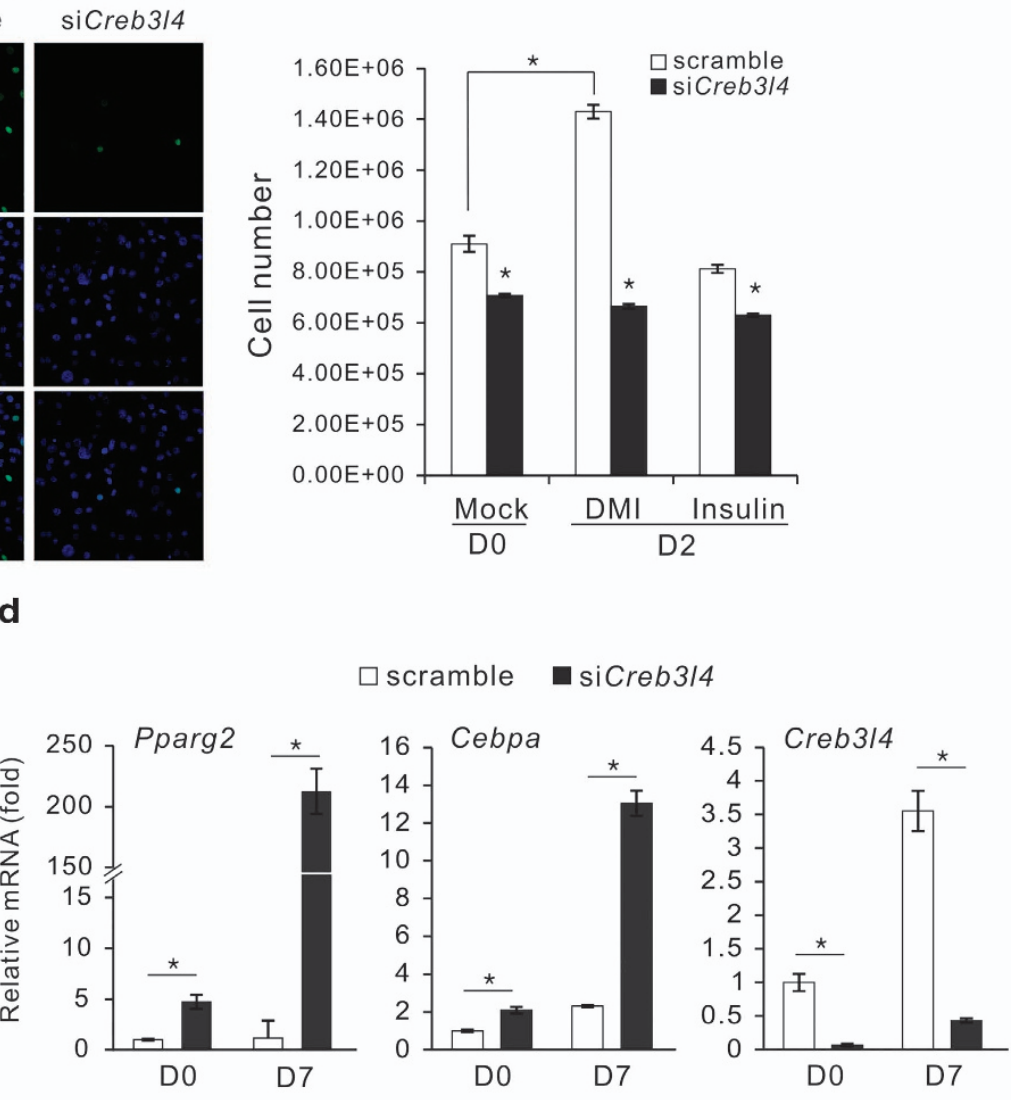

Figure 4 3T3-L1 cells transfected with siCreb3/4 bypass the mitotic clonal expansion step. (a) Immunofluorescence analysis of BrdU-labeled 3T3-L1 adipocytes. The cells were treated with DMI or insulin and were then subjected to BrdU pulsing for $2 \mathrm{~h}$ (from 16 to $18 \mathrm{~h}$ after initiation of differentiation). The period of the BrdU pulse corresponds to the $S$ phase in induced cells. Representative images of fluorescence microscopy ( $\times 40$ magnification). (b) Cell counts were determined at days 0 and 2 after induction of differentiation with $10 \%$ FBS-DMEM containing DMI or insulin $(1 \mu \mathrm{g} / \mathrm{ml})$ after transfection of siCreb3/4. (c) Modified protocol for deletion of mitotic clonal expansion step. Induction of adipogenesis using the modified protocol in siCreb3/4-expressing 3T3-L1 cells treated with insulin (1 $\mu \mathrm{g} / \mathrm{ml})$-containing media (10\% FBS-DMEM) for 2 days, and changed media every 2 days. After 8 days, the cells were stained with oil-red O, and microscopy (×20) was performed. (d) The mRNA levels of Pparg2, Cebpa, and Creb3/4 are determined by real-time PCR. Error bars represent mean \pm S.E.M., $n=3$ per group, ${ }^{*} P<0.05$

CREB3L4 increases the expression of Gata3 and regulates protein stability of C/EBP $\beta$. As shown in Figures $3 c, f$, and g, PPAR 2 and C/EBPa levels were already elevated on D0. Thus, we assumed that elevated expression of PPARy and C/ $\mathrm{EBPa}$ in 3T3-L1 preadipocytes causes differentiation into mature adipocytes. Then, we questioned why PPAR 2 and C/ $\mathrm{EBP} a$ were already increased at this time point. We examined the possible mechanisms of action of CREB3L4, including (1) a direct effect of CREB3L4 on the expression of Pparg2, Cebpa, and Cebpb as a repressor; (2) induction of expression of negative regulators by CREB3L4, which in turn would repress Pparg2 and Cebpa expression; and (3) regulation of C/EBP $\beta$ stability, which would affect Pparg2 and Cebpa expression (Figures $2 \mathrm{~b}$ and $\mathrm{c}$ ). To answer this question, we measured the effect of CREB3L4 on the promoter activities of Pparg2, Cebpa, and Cebpb, which turned out to have no effect (Supplementary Figure S4a). Furthermore, the mRNA levels of most negative regulators ${ }^{7}$ in the Creb3/4-overexpressing group were not altered (Supplementary Figure S4b). However, the expression of Gata3, an important negative regulator in preadipocyte- adipocyte transition, ${ }^{16}$ was induced in the Creb3/4-transduced cells (Figures 2b, c, 5a, and b). Finally, the protein, but not the mRNA, levels of C/EBP $\beta$ decreased in Creb3/4expressing 3T3-L1 cells at D0 (Figures $2 b, 2 c, 5 a$, and $5 b$ ). In siCreb3/4-treated cells, the protein levels of PPARy2, C/ $\mathrm{EBP} \alpha$, and C/EBP $\beta$ also increased at D0 (Figure 5c). However, real-time PCR showed no difference in the Cebpb mRNA levels (Figure $5 \mathrm{~d}$ ). These data suggested that CREB3L4 may act as a negative regulator of the differentiation of 3T3-L1 preadipocytes both by upregulating Gata3 expression and by decreasing the stability of C/EBP $\beta$ protein at D0.

CREB3L4 induces the C/EBP $\beta$ ubiquitination through protein-protein interaction. To examine the effect of CREB3L4 on the stability of C/EBP $\beta$, we treated Creb3/4transfected 3T3-L1 cells with the proteasomal inhibitor, MG132. The Creb3/4-mediated decrease in C/EBP $\beta$ levels was restored in cells treated with MG132 (Figure 6a). Moreover, CREB3L4 induced the ubiquitination of C/EBP $\beta$ (Figure 6b). Because both CREB3L4 and C/EBP $\beta$ have a 
a

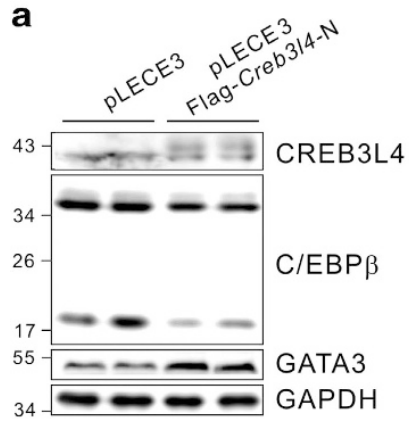

C

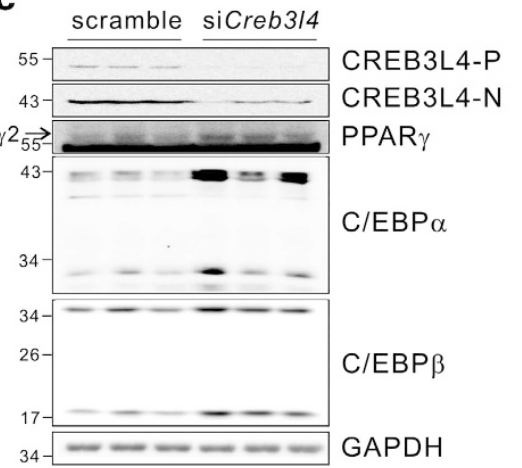

b
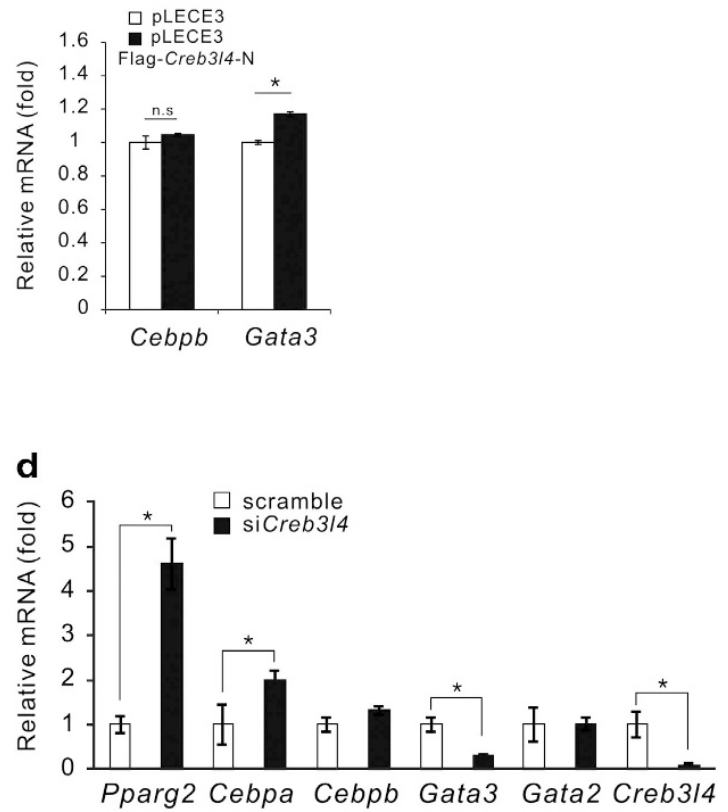

Figure 5 CREB3L4 regulates the stability of C/EBP $\beta$ protein and Gata3 expression. (a, b) Immunoblot and real-time PCR analysis of C/EBP $\beta$ and GATA3 expression in 3T3L1 cells with lentivirus-mediated Creb3/4 expression at D0. (c) Immunoblot of adipogenic factors at D0 in 3T3-L1 cells transfected with siCreb3/4. (d) The mRNA levels of transcription factors at D0 in 3T3-L1 cells transfected with siCreb314. Values are expressed as mean \pm S.E.M., $n=3$ per group, ${ }^{*} P<0.05$

bZIP domain, we performed an immunoprecipitation assay to investigate their interaction. As expected, CREB3L4 interacted with C/EBP $\beta$ (Figure 6c; Supplementary Figure S5a), and their interaction was confirmed using reciprocal antibodies (Figure 6d). Endogenous C/EBP $\beta$ also interacted with CREB3L4 in 3T3-L1 cells (Supplementary Figure S5b). Subcellular fractionation of cells overexpressing the nuclear form of $C R E B 3 L 4^{13}$ and $C / E B P \beta$ revealed that this protein complex is colocalized in the nucleus (data not shown). Interaction between CREB3L4 and C/EBP $\beta$ occurs through their bZIP domains (Figure 6e), suggesting that CREB3L4 induced ubiquitination of $\mathrm{C} / \mathrm{EBP} \beta$ at DO through proteinprotein interaction.

C/EBP $\beta$ mediates upregulation of PPAR 2 by inhibition of Creb314. We next determined whether C/EBP $\beta$ could regulate expression of Pparg2 and Cebpa at D0. 3T3-L1 cells transfected with $\mathrm{Cebpb}$ or Cebpb/Creb3/4 were treated with insulin-containing media every 2 days. Cebpb-transfected cells were differentiated, which was inhibited by cotransfection with Creb3/4 (Figure 7a; Supplementary Figure S6a). Expression of PPARy2 and C/EBPa was increased by transfection with $C e b p b$, but their expression was suppressed by co-transfection with Creb3/4 at D0 (Figure 7b; Supplementary Figure S6b). These data are supported by previous reports demonstrating that ectopic expression of Cebpb in NIH-3T3 cell induced Pparg expression with accompanying adipogenesis. ${ }^{17}$ At D0, stabilized C/EBP $\beta$ in siCreb3/4 group directly bound to the C/EBP binding region of Cebpa and Pparg2 gene promoter (Figures 7c and d).
To investigate the interrelationship between C/EBP $\beta$ and CREB3L4 in the induction of PPARY2 and C/EBPa, we transfected 3T3-L1 cells with siCebpb and siCreb3/4. The cells that had been transfected with siCreb3/4 showed increased adipocyte differentiation, which was inhibited by siCebpb (Figure 7e; Supplementary Figure S6c). In preadipocytes, siCreb3/4-mediated induction of PPARy2 was decreased by Cebpb knockdown (Figure 7f), suggesting that C/EBP $\beta$ is a mediator of siCreb3/4-induced PPARy2 expression in preadipocytes. Because a siCebpb-mediated decrease in PPARy2 protein levels was difficult to observe at D0, we performed quantitative real-time PCR to investigate Pparg2 mRNA levels after treatment with siCebpb. Cebpb knockdown resulted in decreased Pparg2 expression, even at D0 (Supplementary Figure S6d). The siCreb3/4-mediated increase in Pparg2 mRNA levels was also decreased by siCebpb (Supplementary Figure S6d). The expression pattern of Cidec, a PPARytarget gene, was similar to that of Pparg2 (Supplementary Figure S6d).

GATA3 also mediates suppression of Creb3/4-induced PPARy2 regulation. Furthermore, because GATA3 is known to be a negative regulator of Pparg2 expression, ${ }^{16}$ it is possible that downregulation of GATA3 expression by siCreb3/4 (Figure $5 d$ ) could also contribute to the upregulation of PPAR 2 at D0. To test this hypothesis, we transfected 3T3-L1 cells with siGata3; we observed increased expression of PPARy2, but not that of C/EBPa (Figure 8a). In addition, the siCreb3/4-mediated increase in Pparg2 expression was diminished by overexpression of Gata3 (Figure 8b). These data suggested that GATA3 is another mediator upregulating Pparg2 expression when 
a

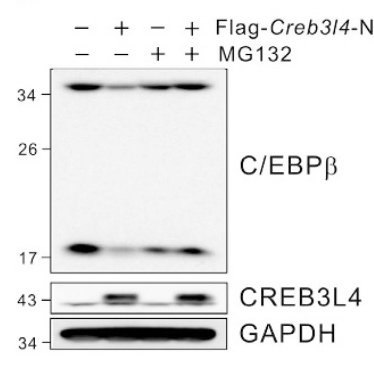

b

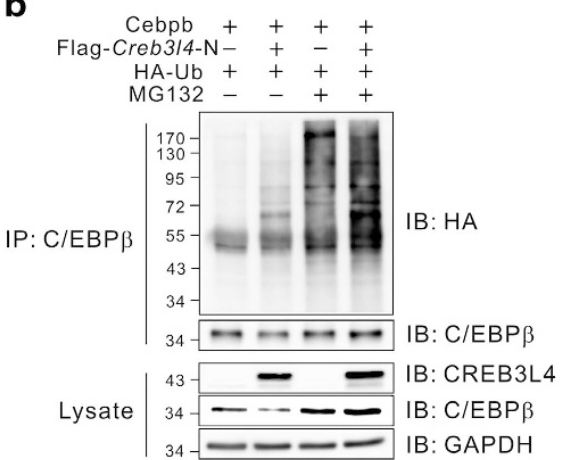

e

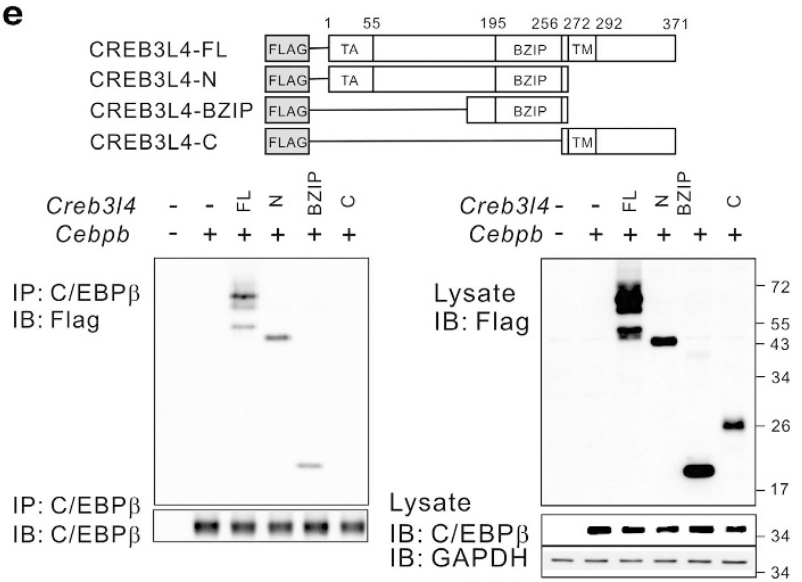

Figure 6 CREB3L4 induces the C/EBP $\beta$ ubiquitination through direct interaction. (a) 3T3-L1 cells were transfected with a Creb3/4-N expression vector and treated with MG132 $(10 \mu \mathrm{M})$ for $4 \mathrm{~h}$. C/EBP $\beta$ expression at day 0 (D0) was detected by immunoblotting. (b) NIH-3T3 cells were co-transfected with Creb3/4, Cebpb, and ubiquitin expression vectors and then treated for $4 \mathrm{~h}$ with MG132 $(10 \mu \mathrm{M})$. Subsequently, an immunoprecipitation assay was carried out. Ubiquitin-conjugated C/EBP $\beta$ was detected by immunoblotting. (c) NIH-3T3 cells transfected with Cebpb and Creb3/4 expression vectors were immunoprecipitated using an anti-C/EBP $\beta$ antibody, or (d) their reciprocal antibodies. (e) Mapping of the CREB3L4 domain interacting with C/EBP $\beta$. Each domain of CREB3L4 is shown in the upper panel. NIH-3T3 cells were transfected with Creb3/4FL, Creb3/4-N, Creb3/4-BZIP, and Creb3/4-C with Cebpb. After incubation for $48 \mathrm{~h}$, cell lysates were immunoprecipitated with anti-C/EBP $\beta$ antibody and probed with anti-Flag antibody

Creb3/4 expression is knocked down. Taken together, these results indicated that $C R E B 3 L 4$ acts as a negative regulator that blocks adipogenesis at D0, by inducing both ubiquitination of $\mathrm{C} / \mathrm{EBP} \beta$ and expression of Gata3.

Creb314-KO mice showed improved metabolic parameters due to increased adipogenesis. To further confirm the effects of CREB3L4 in adipogenesis, adipocyte differentiation in Creb3/4 KO and WT mouse embryonic fibroblasts (MEFs) was compared. Primary Creb3/4 KO MEFs showed an increase in lipid accumulation and morphological differentiation compared with WT MEFs at D22, even in the insulincontaining medium (Figure 9a). To elucidate the physiological role of Creb3/4 in adipogenesis, both WT and Creb3/4-KO mice were fed with a $45 \%$ high-fat diet (HFD) for up to 16 weeks. There are no significant differences in body weight and fat mass (data not shown). However, the size of adipocyte fat cells in Creb3/4-KO mice was smaller than that in WT mice (Figures $9 \mathrm{~b}$ and $\mathrm{c}$ ). The average of epididymal adipocyte diameter in WT mice was $184 \mu \mathrm{m}$, in the Creb3/4$\mathrm{KO}$ mice, average of adipocyte size was smaller $(151 \mu \mathrm{m})$ (Figure 9c). In addition, Creb3/4-KO mice exhibited an increased number of these small adipocytes (hyperplasia), which may contribute to improved glucose tolerance and insulin sensitivity (Figures 9d and e). HFD-fed Creb3/4-KO mice showed decreased fasting glucose levels (Figure 9d) and were resistant to diet-induced hyperinsulinemia (Figure 9f). However, plasma lipid profiles, including triglyceride, total cholesterol, and non-esterified free fatty acid levels, were not significantly different between these groups (Supplementary Figure S7a). Because adipose tissue can affect systemic glucose homeostasis by secreting adipokines, we measured adipokine levels. We found lower levels of leptin, resistin, and PAI-1 in Creb3/4-KO mice than in WT mice, suggesting that deletion of Creb3/4 led to an altered adipokine profile that may contribute to improved insulin sensitivity (Figure 9f). In addition, insulin-stimulated Akt phosphorylation (S473) was augmented in WAT and liver tissues, but not in muscle, of the Creb3/4-KO mice (Supplementary Figure S7b). These results indicate that adipocyte hyperplasia due to increased adipogenesis in HFD-fed Creb3/4-KO mice could be an adaptation to overcome glucose intolerance and insulin resistance. 
a

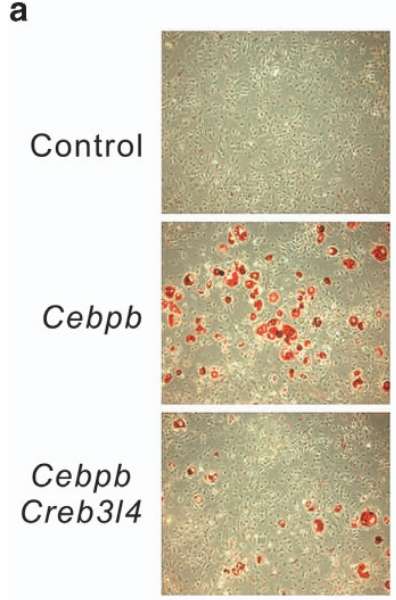

C

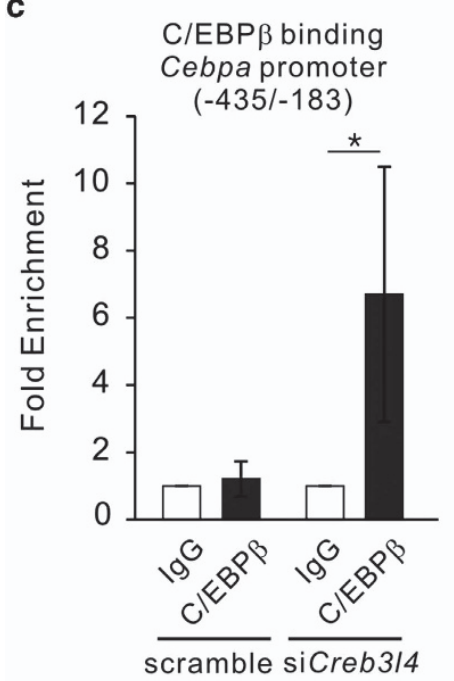

e
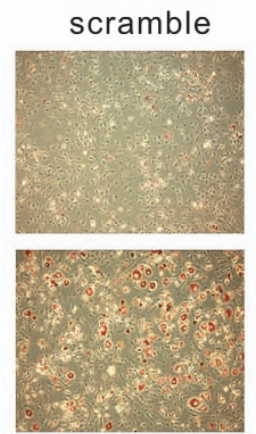

siCreb3/4
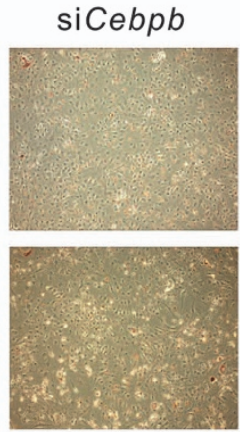

siCebpb siCreb3/4 b

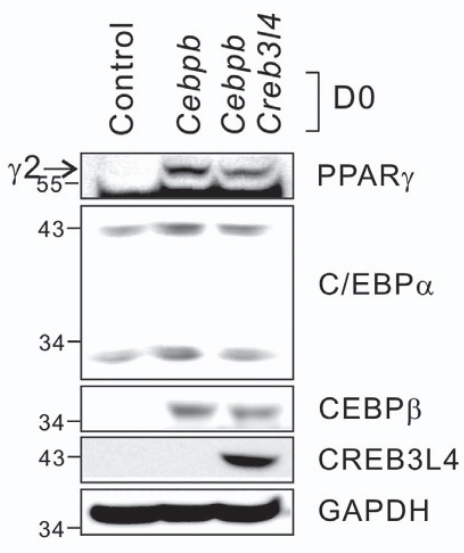

d

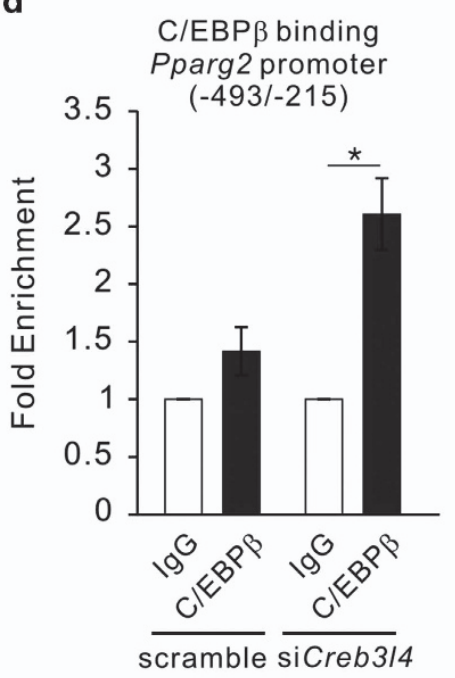

f

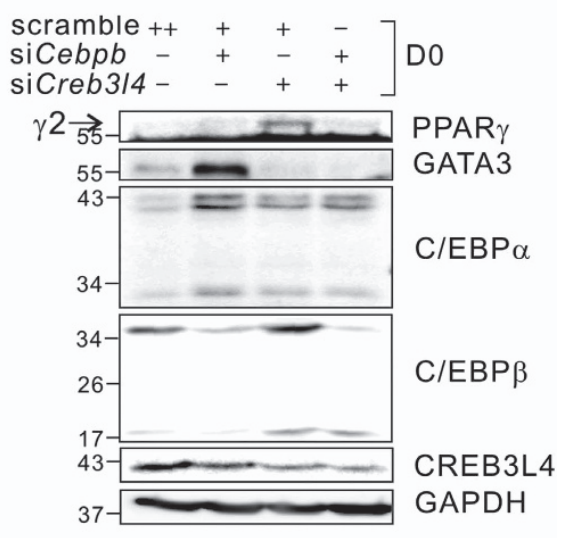

Figure $7 \mathrm{C} / \mathrm{EBP} \beta$ is a mediator of CREB3L4 downregulating the expression of PPAR $\gamma 2$. (a) Oil-red $\mathrm{O}$ staining of adipocytes was performed at $\mathrm{D} 8$, and microscopy ( $\times 20)$ was performed. 3T3-L1 cells were transfected with Cebpb or Cebpb/Creb3/4 and stimulated to differentiate with insulin ( $1 \mu \mathrm{g} / \mathrm{ml})$-containing media (10\% FBS-DMEM) every 2 days. (b) Immunoblot of adipogenic transcription factors in 3T3-L1 cells expressing Cebpb or Cebpb/Creb3/4 at D0. ChIP assay performed in 3T3-L1 cells that transfected with siCreb3/4 at D0. Normal IgG was used as a negative control for immunoprecipitation. The regions amplified were -435 to -183 bp for Cebpa gene promoter (c) and -493 to -215 for Pparg2 gene promoter (d). The quantity of immunoprecipitated DNA was normalized to total input DNA. Values are expressed as mean \pm S.E.M., $n=3$ per group, ${ }^{*} P<0.05$. (e) Oil-red O staining of 3T3-L1 cells transfected with siCebpb and siCreb3/4 at D8, and microscopy (×20) was performed. The cells were stimulated to differentiate as described in (a). (f) Immunoblot of adipogenic factors in 3T3-L1 cells expressing siCebpb and siCreb3/4, at DO 

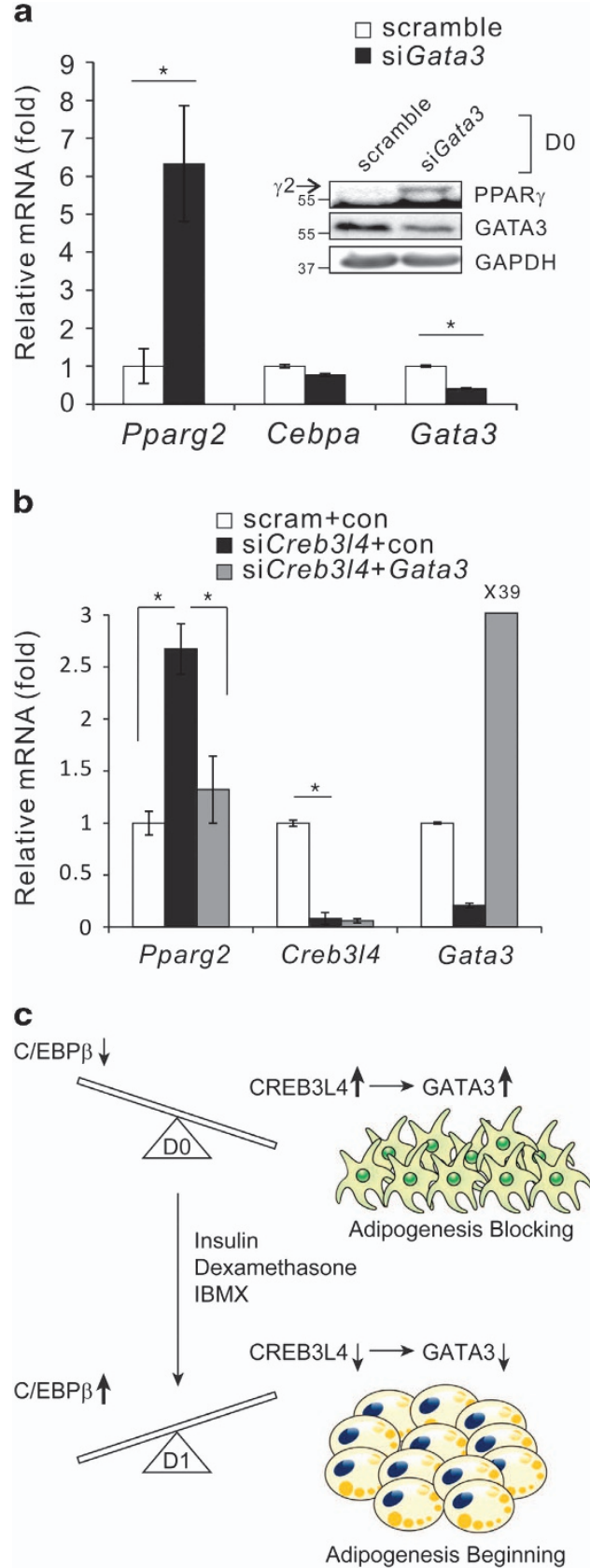

Figure 8 GATA3 is another mediator of CREB3L4 downregulating the expression of PPAR $\gamma 2$. (a) Expression of PPAR $\gamma 2$ and C/EBP $\alpha$ in 3T3-L1 cells expressing siGata3, at D0. (b) The mRNA levels of Pparg2, Creb3/4, and Gata3 in 3T3-L1 cells transfected with siCreb3/4 and a CMV-mGata3-FL expression vector, at D0. Values are expressed as mean \pm S.E.M., $n=3,{ }^{*} P<0.05$. (c) In preadipocytes, CREB3L4 acts as a negative regulator of adipogenesis by both regulating the stability of C/EBP $\beta$ protein and increasing GATA3 expression. After induction of differentiation by DMI (DMI: $0.5 \mathrm{mM}$ 3-isobutyl-1-methylzanthine [IBMX; M], $1 \mu \mathrm{M}$ dexamethasone [D], and $1 \mu \mathrm{g} / \mathrm{ml}$ of insulin [l]), Creb3/4 and Gata3 expression is decreased, and increased C/ $\mathrm{EBP} \beta$ levels initiate adipogenesis by increasing the expression of PPAR 2 and $\mathrm{C} /$ EBP $\alpha$. Suppression of $\mathrm{Creb} 3 / 4$ in preadipocytes induces adipogenesis by increasing PPAR 2 expression

\section{Discussion}

Adipose tissue serves as an important regulator of metabolic homeostasis, and thus, accumulation of fat depots is associated with insulin resistance and a high risk for type 2 diabetes and metabolic syndrome. ${ }^{18}$ Studying the biological networks and transcription factors, especially negative regulators, that control adipogenesis is important in the development of a therapeutic strategy for obesity-associated type 2 diabetes and metabolic syndrome. In the present study, we explored the role of CREB3L4 in adipogenesis and defined a novel mechanism regulating initiation of adipogenesis. Suppression of Creb3/4 resulted in the expression of PPAR 2 and $\mathrm{C} / \mathrm{EBP} a$, followed by initiation of adipogenesis even in the presence of minimal hormonal inducer in 3T3-L1 preadipocytes, and induced hyperplastic WAT with the improved metabolic parameters.

Although there was a difference in the degree of adipocyte differentiation depending on the stimuli used, viz., insulin or dexamethasone, adipogenesis did occur in all of the siCreb3/4-treated groups (Figure 3b; Supplementary Figure $\mathrm{S} 3 \mathrm{~b})$. Because insulin is known to be a strong inducer of lipogenic gene expression, insulin treatment of siCreb3/4- and shCreb3/4-transfected cells resulted in accelerated adipogenesis, as compared with the mock- or dexamethasone-treated group. Insulin strongly induces the expression of Add1/ Srebp1, which, in turn, promotes adipocyte differentiation by augmenting PPARy-mediated gene expression. ${ }^{19}$ Indeed, ADD1/SREBP1 and PPARy were shown to cooperate to produce PPAR $y$ ligand and to stimulate Pparg expression. ${ }^{20,21}$ These data suggested that CREB3L4 could be a critical negative regulator determining preadipocyte-adipocyte transition in 3T3-L1 cells and that knockdown of this gene could promote adipogenesis.

At D0, it seemed that elevated expression of PPARy and C/ EBPa, by suppression of Creb3/4, resulted in transition of 3T3L1 preadipocytes to mature adipocyte. These data are supported by previous reports that demonstrated that retroviral expression of Pparg2 induced differentiation of cultured fibroblasts into adipocytes, by cooperating with C/EBPa in stimulating the adipocyte program. Moreover, PPARy alone can initiate the entire adipogenesis program in adipocytes. ${ }^{7,22}$ Thus, it is likely that elevation of PPARy2 and C/EBPa due to Creb3/4-knockdown could initiate adipogenesis by sequentially inducing expression of genes that are involved in adipocyte differentiation, even in the presence of minimal hormonal inducer.

The increase in PPARy2 and C/EBPa expression may have further significance. Inhibition of Creb3/4 in 3T3-L1 preadipocytes decreased BrdU incorporation and resulted in a lack of further increase in cell number after treatment with DMI or insulin alone, which seemed to involve increased expression of C/EBPa and PPARy2. C/EBPa has anti-mitotic and antiproliferative actions in terminally differentiating adipocytes and myeloid cells. ${ }^{23,24}$ Ectopic and endogenous expression of Pparg is also sufficient to cause cell-cycle arrest, by modulating E2F phosphorylation. ${ }^{25}$ Additionally, PPARy and $\mathrm{C} / \mathrm{EBP} a$ can promote growth arrest by upregulating the $\mathrm{Cdk}$ inhibitor. ${ }^{26,27}$ Thus, PPARy2 and C/EBPa expression by siCreb3/4 caused adipocyte differentiation, bypassing the MCE step for adipogenesis.

Our study collectively demonstrates that CREB3L4 tightly controls the preadipocyte to adipocyte transition through a dual mechanism, viz., decreasing protein stability of C/EBP $\beta$ 


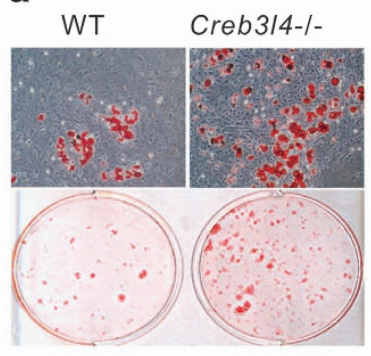

b

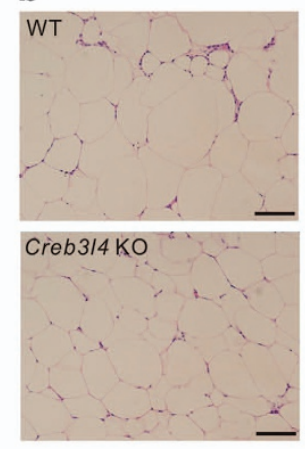

c

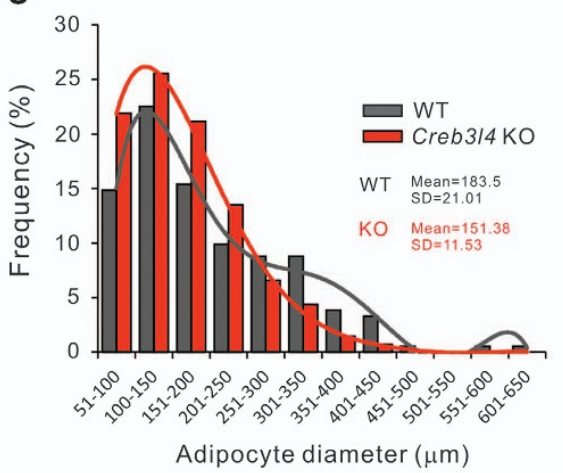

d

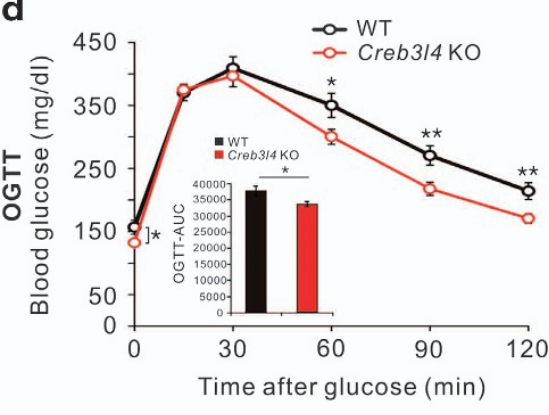

e

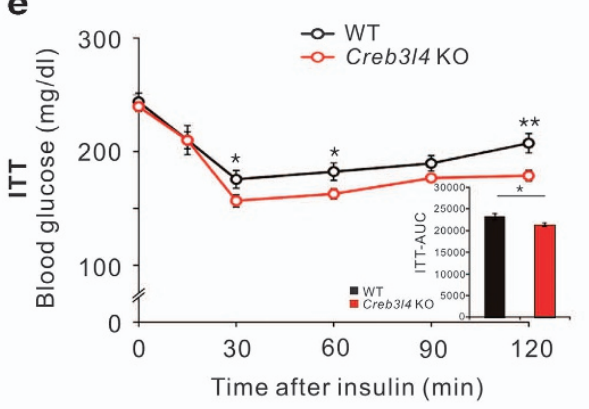

f

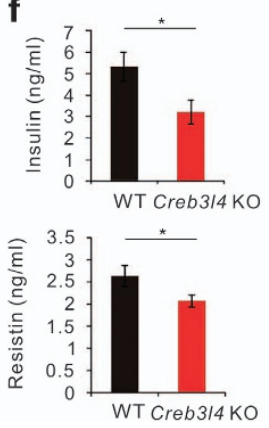

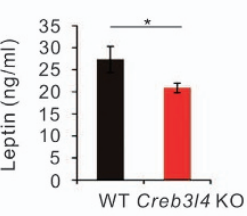

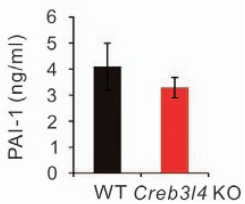

Figure 9 Creb3/4-KO mice showed adipocyte hyperplasia, lead to improved metabolic parameters. (a) Adipogenic potential of mouse embryonic fibroblasts (MEFs) derived from wild-type (WT) or Creb3/4-knockout (KO) mice. Cells were stained with oil-red O after treatment with insulin (10 $\mu \mathrm{g} / \mathrm{ml})$ and rosiglitazone $(2 \mu \mathrm{M})$ for 6 days, followed by insulin $(1 \mu \mathrm{g} / \mathrm{ml})$ for 16 days. Microscopic ( $\times 20)$ views representative of three independent experiments are shown. (b) Hematoxylin and eosin (H\&E) staining of epididymal fat. Scale bar: $100 \mu \mathrm{m}$. (c) Adipocyte diameter was quantified with Image J ( $n=3$ ). (d) Oral glucose tolerance test (GTT), (e) insulin tolerance test (ITT), performed after 14 and 15 weeks, respectively, of feeding HFD $(n=12-19)$. (f) Insulin level from mice was measured by ELISA ( $n=9-14)$. Leptin, resistin, and PAI-1 levels from mice fed HFD for 16 weeks were measured by MAGPIX, using a MILLIPLEX MAP mouse magnetic bead panel $(n=6-12)$. Values are expressed as mean \pm S.E.M., ${ }^{*} P<0.05,{ }^{* *} P<0.01$

through ubiquitination, and upregulation of Gata3 expression. It was unclear why CREB3L4 induces ubiquitination of C/ $\mathrm{EBP} \beta$ at D0. Presumably, because $\mathrm{C} / \mathrm{EBP} \beta$, as a transcription factor, induces expression of downstream adipogenic transcription factors, CREB3L4 may induce C/EBP $\beta$ ubiquitination to maintain low levels of $\mathrm{C} / \mathrm{EBP} \beta$ to retain preadipocyte status. CREB3L4 could induce the ubiquitination of C/EBP $\beta$, but further study, which E3 ligase is involved with CREB3L4, is required to understand the ubiquitin conjugation mechanism for $\mathrm{C} / \mathrm{EBP} \beta$, including ubiquitin ligase. In addition, we observed that knockdown of C/EBP $\beta$ expression also decreased the expression of CREB3L4 (Figure 7f; Supplementary Figure S6d). These data suggest that C/EBP $\beta$ and CREB3L4 are co-regulated with each other. Thus, there may exist positive or negative transcription factors regulating the expression of C/EBP $\beta$ and CREB3L4, respectively. Because the regulation of CREB3L4 during differentiation is not known at present, molecular mechanism(s) of how C/EBP $\beta$ downregulates CREB3L4 need to be explored. Further studies on the interplay between C/EBP $\beta$ and CREB3L4 may help understand their role in the adipocyte differentiation. In addition, GATA3 expression is decreased by treatment of siCreb3/4 in 3T3-L1 preadipocytes, resulting in upregulation of PPARY2, but not of C/EBPa (Figure 8a), which indicates that GATA3 affects only Pparg2 expression. These data are consistent with a previous report that GATA3 acts as a negative regulator of adipocyte differentiation through inhibition of Pparg expression, by directly binding to the Pparg2 promoter. ${ }^{16}$ Gata3-deficient ES cells also showed enhanced capacity of adipocyte differentiation under minimally permissive hormonal condition. ${ }^{16}$ Our study suggests that CREB3L4 could be upstream factor for Gata3 gene expression in preadipocytes. In addition, GATA3 is capable of forming protein complexes with either $\mathrm{C} / \mathrm{EBP} a$ or $\mathrm{C} / \mathrm{EBP} \beta$, resulting in the suppression of adipocyte differentiation. ${ }^{28}$ Because GATA3, C/EBPa/ $\beta$, and CREB3L4 are closely associated with the initiation of adipogenesis, the interrelationship among these molecules needs to be identified in detail to understand the initiation of adipogenesis.

In general, adipose tissue expands by increased adipocyte size (hypertrophy) and number (hyperplasia). ${ }^{29}$ However, it is known that HFD-induced adipose tissue expansion is triggered mainly by hypertrophy during the first month of HFD feeding. After prolonged HFD exposure (i.e., longer than 1 month), Epi-WAT showed a high adipogenesis rate as determined by a large number of new fat cells (hyperplasia). ${ }^{30}$ Differentiated adipocytes are post-mitotic; therefore, hyperplasia represents an increase in de novo adipocyte formation (adipogenesis). Creb3/4-KO mice showed increased adipogenesis compared with WT littermates (Figures $9 \mathrm{~b}$ and $\mathrm{c}$ ), similar to the findings observed in 3T3-L1 cells. Consequentially, HFD-fed Creb3/4-KO mice exhibited improved metabolic parameters due to the increase in adipocyte number (Figures 9d-f). 
However, the phenotype of the Creb3/4 KO mice is mild. Because Creb3/4 is expressed in other tissues, the generation and characterization of adipocytes-specific knockout mice will be required to determine the roles of CREB3L4 in obesity and related metabolic diseases.

Taken together, we demonstrated a role for CREB3L4 in the differentiation of 3T3-L1 preadipocytes, which functions as a negative regulator, blocking adipogenesis. In these preadipocytes, CREB3L4 increased the expression of Gata3 as well as the ubiquitination of $\mathrm{C} / \mathrm{EBP} \beta$, to retain the preadipocyte status of the cells. When the cells were exposed to adipogenic inducers (DMI), Creb3/4 expression was decreased, leading to commencement of adipogenesis. It is likely that Creb3/4 expression determines the fate of cells to differentiate into adipocytes (Figure 8c). Furthermore, CREB3L4 and C/EBP $\beta$ genes express contrariwise during the differentiation process $^{31}$ and to the component of the differentiation cocktail (Figure 1d). Turning off of CREB3L4 expression is a prerequisite to turning on the $\mathrm{C} / \mathrm{EBP} \beta$ expression. Thus, it is speculated that a balance between these proteins is critical for adipocyte differentiation (Figure 8c). Indeed, Creb3/4-KO mice subjected to an HFD showed increased hyperplastic WAT, due to enhanced adipogenesis, which may contribute to overcome HFD-induced glucose intolerance and insulin resistance. These results raise the possibility that $C r e b 3 / 4$ could be a useful therapeutic target in the fight against obesity and metabolic syndrome.

\begin{abstract}
Materials and Methods
Animal experiments and KO mice. SVF and AdipoF were isolated from epididymal fat pads of 12-week-old C57BL/6J (C57BL/6JJmsSlc) mice. Briefly, the fat pads were rapidly excised, finely minced, and incubated at $37^{\circ} \mathrm{C}$ for $1 \mathrm{~h}$ with $1 \mathrm{mg} / \mathrm{ml}$ type I collagenase in Hank's buffered salt solution containing $1 \%$ BSA, $200 \mathrm{nM}$ adenosine, and $50 \mathrm{mg} / \mathrm{ml}$ glucose. After sequential filtration and centrifugation, floating adipocytes (AdipoF) and pellet (SVF) were separated. ${ }^{32}$ Creb3/4-KO mouse embryos were purchased from RIKEN (The Institute of Physical and Chemical Research, Ibaraki, Japan) with permission from Dr Nojima. The Creb3/4-KO mice have been described previously. ${ }^{10}$ At 11 weeks of age, Creb3/4KO mice and their WT littermates were fed a 45\% HFD (Research Diets, New Brunswick, NJ, USA) for 18 weeks. To test activation of insulin signaling pathway, mice fed HFD were fasted for $6 \mathrm{~h}$ and then received insulin $(5 \mathrm{U} / \mathrm{kg})$ by intraperitoneal injection. After $5 \mathrm{~min}$, the mice were killed. $^{33,34}$ All the animal experiments were approved by the Institutional Animal Care and Use Committee of Yonsei University College of Medicine.
\end{abstract}

Glucose tolerance and insulin tolerance tests. For oral glucose tolerance tests, mice were fasted for $16 \mathrm{~h}$ and then challenged with oral gavage of glucose ( $2 \mathrm{~g} / \mathrm{kg}$ body weight). For insulin tolerance tests, mice were fasted for $6 \mathrm{~h}$ and then given an intraperitoneal injection of insulin $(0.75 \mathrm{U} / \mathrm{kg}$ body weight; Humulin R, Eli Lilly, Indianapolis, IN, USA). Blood glucose levels were measured, by using a glucose monitor (One TOUCH Sure Step, Life Scan, Milpitas, CA, USA), in blood drawn from the tail vein. The area under the curve of glucose was calculated during the course of the tests.

Phenotypic evaluation of mice. Plasma insulin levels were then measured using ELISA kits (ALPCO Immunoassays, Salem, NH, USA). Plasma adipokines were measured using MAGPIX (Luminex, Austin, TX, USA) with MILLIPLEX MAP mouse magnetic beads (Merck Millipore Corp., St Charles, MO, USA). Other plasma metabolites, such as cholesterol, triacylglycerol, and NEFA were measured by an enzymatic method using an autoanalyzer (Hitachi 7600; Hitachi Instruments, Tokyo, Japan) as per the manufacturer's instructions.

Cell culture and induction of differentiation. 3T3-L1 preadipocytes were cultured in DMEM containing $10 \%$ calf serum (10\% CS-DMEM), $100 \mathrm{U} / \mathrm{ml}$ penicillin, $100 \mu \mathrm{g} / \mathrm{ml}$ streptomycin, and $8 \mu \mathrm{g} / \mathrm{ml}$ biotin, until they reached confluence, and then the cells were maintained for an additional 2 days in the same medium. These post-confluent cells (day 0 [D0]) were stimulated to differentiate using DMEM containing $10 \%$ fetal bovine serum (10\% FBS-DMEM), and a mixture of $0.5 \mathrm{mM} \mathrm{3-}$ isobutyl-1-methylxanthine, $1 \mu \mathrm{M}$ dexamethasone, and $1 \mu \mathrm{g} / \mathrm{ml}$ of insulin (DMI) for 2 days. Cells were then further maintained for 2 days in $10 \%$ FBS-DMEM containing only $1 \mu \mathrm{g} / \mathrm{ml}$ of insulin. After D4, the media were replaced every other day with $10 \%$ FBS-DMEM without insulin. To suppress differentiation in the scramble group, the adipocyte differentiation protocol was modified. Briefly, the cells were incubated with $10 \%$ FBS-DMEM containing mock (no treatment), insulin $(1 \mu \mathrm{g} / \mathrm{ml})$, or dexamethasone $(1 \mu \mathrm{M})$, respectively, from $\mathrm{D} 0$, and media were changed every 2 days until D8, while maintaining the respective hormonal stimulation. For MEF studies, heterozygous animals were bred to generate E13.5 embryos. Primary MEFs (passages 1-2) obtained from E13.5 embryos were grown to confluency (D0) in $10 \%$ FBS-DMEM. Adipocyte differentiation was induced by treating cells with $10 \%$ FBS-DMEM containing insulin $(10 \mu \mathrm{g} / \mathrm{ml})$ and rosiglitazone $(2 \mu \mathrm{M})$ for 6 days. Thereafter, the cells were maintained in 10\% FBS-DMEM containing only insulin $(1 \mu \mathrm{g} / \mathrm{ml})$ for 16 days.

Total RNA isolation and quantitative real-time PCR. Total RNA was isolated from mouse tissues using TRlzol reagent (Invitrogen, Carlsbad, CA, USA), and total RNA of 3T3-L1 cells was isolated using the Easy Spin RNA extraction kit (iNtRON, Gyeonggi-do, South Korea) according to the manufacturer's instructions. This RNA was used to generate cDNA using the ImProm-II Reverse Transcription System (Promega, Madison, WI, USA). Quantitative real-time PCR (qPCR) was performed using the Step One Real-Time PCR Systems instrumentation and software (Applied Biosystems, Foster City, CA, USA) according to the manufacturer's protocol. The relative amount of mRNA in each sample was normalized to Rplpo (36B4) transcript levels. The sequences for the gene-specific PCR primers are listed in Supplementary Table S1.

Western blotting. Proteins from 3T3-L1 and NIH-3T3 cells were isolated using the Pro-Prep protein extraction solution (iNtRON) containing appropriate protease inhibitors. The lysate was heated at $100^{\circ} \mathrm{C}$ for $10 \mathrm{~min}$, centrifuged for $5 \mathrm{~min}$ at 13000 r.p.m. to remove the cell debris. Protein concentration was determined using BCA protein assay (Thermo Scientific, Rockford, IL, USA). The protein $(50 \mu \mathrm{g})$ in each lane was subjected to sodium dodecyl sulfate-polyacrylamide gel electrophoresis (SDS-PAGE) and transferred onto nitrocellulose membranes (Whatman, Dassel, Germany). Membranes were blocked with $5 \%$ non-fat milk and incubated with the following primary antibodies: anti-CREB3L4 (AT1618a, Abgent Inc., San Diego, CA, USA), anti-C/EBP $\beta$ (sc-150), anti-C/EBP $\alpha$ (sc-61), (Santa Cruz Biotechnology Inc., Santa Cruz, CA, USA), anti-AKT (pan) (\#4691s), anti-p-AKT (\#9271s), anti-GAPDH (\#2118), anti-PPAR $\gamma$ (\#2443), anti-p-C/EBP $\beta$ (\#3084) (all from Cell Signaling, St Louis, MO, USA), anti-GATA3 (ab106625, Abcam, Cambridge, MA, USA), anti-HA (\#11583816001, Roche, Mannheim, Germany), and anti-Flag (F3165, Sigma-Aldrich, St Louis, MO, USA), and anti-LXR ${ }^{35}$ antibodies. Membranes were then incubated with anti-mouse or anti-rabbit goat horseradish peroxidase-conjugated secondary antibodies (Thermo Scientific) at a $1: 4000$ dilution, in $5 \%$ non-fat milk in PBST buffer $(137 \mathrm{mM} \mathrm{NaCl}, 2.7 \mathrm{mM} \mathrm{KCl}$, $10 \mathrm{mM} \mathrm{Na}_{2} \mathrm{HPO}_{4}, 2 \mathrm{mM} \mathrm{KH}_{2} \mathrm{PO}_{4}, 0.1 \%$ Tween-20), for $1 \mathrm{~h}$ at room temperature. Target proteins were visualized using an enhanced chemiluminescence detection system (West Pico \& West Dura, Thermo Scientific). The protein bands were detected using a Fujifilm LAS-3000 Imager (FUJIFILM Corporation, Tokyo, Japan).

Ubiquitination assay. Cells were transfected with Flag-tagged Creb3/4 and HA-tagged ubiquitin using FuGENE HP transfection reagent (Promega Corporation). After $48 \mathrm{~h}$, cells were treated with $10 \mu \mathrm{M} \mathrm{MG} 132$ for $4 \mathrm{~h}$ before harvesting, and were then resuspended in cell lysis buffer $(50 \mathrm{mM}$ Tris $(\mathrm{pH} 7.4), 150 \mathrm{mM} \mathrm{NaCl}, 0.2 \%$ Triton X-100, 0.3\% NP-40) containing protease-inhibitor cocktail tablets (Roche). The lysates were centrifuged and precleared with protein G-agarose (\#05015952001, Roche), followed by a 2-h incubation with anti-C/EBP $\beta$ antibody (Santa Cruz Biotechnology Inc.) and a 1-h incubation with protein G-agarose at $4^{\circ}$ C. Beads were washed twice with washing buffer (1/3 dilution of cell lysis buffer in phosphate-buffered saline (PBS)), and briefly centrifuged and resuspended in the sample buffer before being subjected to SDS-PAGE.

Immunoprecipitation assay. Constructs expressing Flag-tagged Creb3/4 and Cebpb were co-transfected into NIH-3T3 cells using FuGENE HP transfection reagent. After $48 \mathrm{~h}$, cells were harvested and lysed in cold cell lysis buffer $(50 \mathrm{mM}$ 
Tris ( $\mathrm{pH} 7.4$ ), $150 \mathrm{mM} \mathrm{NaCl}, 0.2 \%$ Triton X-100, 0.3\% NP-40) containing appropriate protease inhibitors. Whole-cell lysate $(800 \mu \mathrm{g})$ was precleared with protein G-agarose (\#05015952001, Roche) followed by incubation with anti-C/EBP $\beta$ antibody or anti-CREB3L4 antibody for $16 \mathrm{~h}$ and protein G-agarose for $2 \mathrm{~h}$ at $4{ }^{\circ} \mathrm{C}$. After centrifugation, the protein $\mathrm{G}$-agarose pellets were washed several times with washing buffer (1/3 dilution of cell lysis buffer in PBS) and resuspended in sample buffer before being subjected to SDS-PAGE.

Small-interfering RNA. RNA oligonucleotides for scramble (forward, $5^{\prime}$-CCU ACGCCACCAAUUUCGUdTdT-3') (Bioneer), mouse Creb3/4 (forward, $5^{\prime}$-UUUCUG AGCAGUGUAUCAUAUUGGG-3'), mouse Gata3 (forward, 5'-CAUGAAGCUGGAG ACGUCUCACUCU-3'), and mouse Cebpb (forward, 5'-AGUAGAAGUUGGCCAC UUCCAUGGG-3') (Invitrogen) were synthesized. Each siRNA $(30 \mathrm{nM})$ was transfected into appropriate experimental sets of 3T3-L1 cells using Lipofectamine RNA iMAX (Invitrogen) for at least $48 \mathrm{~h}$; cells were then lysed for RNA and protein preparation.

Construction of plasmids. Expression constructs encoding the full-length (FL), nuclear (N), BZIP (BZIP), and C-terminal (C) forms of mouse Creb3/4 were PCR amplified and cloned directly into the pcDNA3.1-Flag2 vector. Similarly, the PCR-amplified fragments of Cebpb were inserted into the BamHI/Xhol sites of an expression vector, pcDNA3.1 mCebpb-V5/His. To construct luciferase reporters, mouse Cebpb regions - 1951/+181 and mouse Cebpa regions - 1884/+220 were subcloned into the Kpnl/Xhol sites of pGL4.14. The mouse Pparg2 promoter covering the region $-2000 /+27$ was a generous gift from Dr JW Kim. ${ }^{36}$ CMVmGATA3-FL, encoding the full-length mouse Gata3 was a gift from $\mathrm{Dr}$ GR Lee. ${ }^{37}$ $\mathrm{HA}$-tagged ubiquitin expression vector was a gift from Dr HG Yoon. ${ }^{38} \mathrm{~A}$ lentiviral expression vector, pLECE3-mCreb3/4-N, was constructed by subcloning the mouse nuclear form of Creb3/4 into the Hpal/Notl sites of pLECE3. The lentiviral vector pLECE3 was a kind gift from Dr KH Chun. ${ }^{39}$ The lentiviral vector pLKO.1-shRNACreb3/4 (TRCN0000086183) and pLKO.1 puro Non-Target shRNA were purchased (Sigma-Aldrich). The shRNA sequence of Creb3/4 was as follows: $5^{\prime}$-CCGG;G CACCTCAAATGCTTGTCATACTCGAGTATGACAAGCATTTGAGGTGCTTTTTG-3'.

BrdU incorporation assay. BrdU labeling was performed on cells induced to differentiate on coverslips with DMI or insulin alone, using a 5-Bromo-2'-deoxyuridine Labeling and Detection Kit I (Roche) according to the manufacturer's instructions. The coverslips were subsequently mounted for immunofluorescence analysis.

Establishment of a stable cell line. The lentivirus particles were generated using three plasmids, VSV (pMDG), RSV-REV, and PMDLg/pRRE, in which 293FT cells were co-transfected with pLECE3-Flag-mCreb3/4(N) (controlpLECE3) or pLKO.1-shRNA-Creb3/4 (control-pLKO.1 puro Non-Target shRNA). The 293FT cells were transfected using Plus-Lipofectamine transfection reagent (Invitrogen) and the media was replaced with 10\% CS-DMEM. Two days after transfection, the cell culture media was filtered using a $0.45-\mu \mathrm{m}$ syringe filter. Culture media for 3T3-L1 cells were changed to a $1: 5$ mixture of viral supernatant and fresh $10 \%$ CS-DMEM containing polybrene (H9268, Sigma-Aldrich) at a final concentration of $8 \mu \mathrm{g} / \mathrm{ml}$. After 3 days, lentivirus-infected 3T3-L1 cells were selected using $2 \mu \mathrm{g} / \mathrm{ml}$ of puromycin (Sigma-Aldrich).

Chromatin immunoprecipitation assay. Chromatic immunoprecipitation (ChIP) experiments were performed as previously described ${ }^{35}$ with 3 T3-L1 cells transfected with scramble or siCreb3/4 at DO. Briefly, protein extracts were incubated for $15 \mathrm{~h}$ at $4{ }^{\circ} \mathrm{C}$ with $6 \mu \mathrm{g}$ anti-C/EBP $\beta$ (sc-150, Santa Cruz Biotechnology Inc.) or normal rabbit IgG (sc-2027, SantaCruz Biotechnology Inc.) antibodies. Protein and/or DNA complexes were precipitated for $1 \mathrm{~h}$ at $4{ }^{\circ} \mathrm{C}$ using $60 \mu \mathrm{l}$ of $50 \%$ (vol/vol) protein $\mathrm{G}$ agarose/salmon sperm DNA slurry. DNA fragments were purified using a PCR purification kit (28106; Qiagen, Valencia, CA, USA) and measured by quantitative real-time PCR. All reactions were normalized relative to input activities to account for chromatin sample preparation differences $\left(\Delta \mathrm{C}_{\mathrm{t}}\right)$ and determined to the difference between the $\alpha$-C/EBP $\beta$ IP sample $\left(\Delta \mathrm{C}_{\mathrm{t}[\mathrm{C} / \mathrm{EBP} \beta]}\right)$ and $\alpha$-lgG IP sample $\left(\Delta \mathrm{C}_{\mathrm{t}[\mathrm{lgG}]}\right)$ for fold enrichment. $\left.\Delta \Delta \mathrm{C}_{\mathrm{t}} \mathrm{C} / \mathrm{EBP} \beta-\mathrm{lgG}\right]=\Delta \mathrm{C}_{\mathrm{t}}[\mathrm{C} / \mathrm{EBP} \beta]-\Delta \mathrm{C}_{\mathrm{t}[\mathrm{lgG}]}$, Fold change in occupancy $=2^{(-\Delta \Delta C+[C / E B P \beta-\lg ]])}{ }^{40,41}$ The primers used for the PCR of the promoter of C/EBP binding regions of Pparg2 gene (F-5'-TTCAGATGTGTGATTAGGAG-3', R-5'-AGACTTGGTACATTACAAGG), and Cebpa gene (F-5'-TCCCTAGTGTTGGCTGGAAG-3', R-5'-CAGTAGGATGGTGCC TGCTG-3'). ${ }^{42}$
Electroporation of 3T3-L1 preadipocytes and transient transfection. To maximize the transfection efficiency, 3T3-L1 preadipocytes were transfected using an OneDrop Microporator MP-100 (Digital Bio, Seoul, South Korea). The cells were transfected using a pipette-type Neon Transfection System according to the manufacturer's instruction (MPK10096, Invitrogen). The cells were electroporated at $1300 \mathrm{~V}$, using two pulses with a 20-ms pulse width. NIH-3T3 cells were plated in 12well tissue culture dishes at a density of $2 \times 10^{5}$ cells/well in $1 \mathrm{ml}$ DMEM. Expression plasmids for Creb3/4 (0, 50, 100, and $200 \mathrm{ng})$, luciferase-tagged mPparg2 - 2000/ +27, mCebpb - 1951/+181, mCebpa - 1884/+220 (500 ng), and Renilla luciferase were co-transfected using FuGENE HP Transfection Reagent (Promega) at a ratio of $3: 1$. The total amount of transfected plasmid was adjusted to $200 \mathrm{ng}$ by adding empty vector. All luciferase experiments were performed using a Dual-Luciferase Reporter Assay System (Promega). Luciferase activities were normalized to Renilla luciferase activities to adjust transfection efficiency.

Hematoxylin and eosin staining. Epi-WAT was fixed with 10\% neutralbuffered formalin, embedded in paraffin, and sectioned. Hematoxylin and eosin (H\&E) staining was then performed on these sections.

Statistical analysis. Three to five experiments were performed for in vitro studies, using triplicate replicates of each transfection. The data are represented as means \pm standard error of the mean (S.E.M.). All data of in vitro and in vivo sets were analyzed for statistical significance using non-parametric Mann-Whitney tests and independent samples $t$-Test, respectively. All $P$-values $<0.05$ were considered as significant. Statistical analysis was performed using SPSS (IBM SPSS statistics ver. 20; IBM Corp., Armonk, NY, USA).

\section{Conflict of Interest}

The authors declare no conflict of interest.

Acknowledgements. We thank Dong-Su Jang for the schematic representation of the model of CREB3L4 action. This work was supported by the National Research Foundation of Korea (NRF) grants funded by the Korea government (MSIP) (NRF-2009-0080655 and NRF-2011-0030086 to YHA, NRF-2011-0014431 to THK).

1. Jo J, Gavrilova O, Pack S, Jou W, Mullen S, Sumner AE et al. Hypertrophy and/or hyperplasia: dynamics of adipose tissue growth. PLoS Comput Biol2009; 5: e1000324.

2. Kahn SE, Hull RL, Utzschneider KM. Mechanisms linking obesity to insulin resistance and type 2 diabetes. Nature 2006; 444: 840-846.

3. Lefterova MI, Lazar MA. New developments in adipogenesis. Trends Endocrinol Metab 2009; 20: 107-114.

4. Lin FT, Lane MD. CCAAT/enhancer binding protein alpha is sufficient to initiate the 3T3-L1 adipocyte differentiation program. Proc Natl Acad Sci USA1994; 91: 8757-8761.

5. Lazar MA. Becoming fat. Genes Dev2002; 16: 1-5.

6. Tang QQ, Gronborg M, Huang H, Kim JW, Otto TC, Pandey A et al. Sequential phosphorylation of CCAAT enhancer-binding protein beta by MAPK and glycogen synthase kinase 3beta is required for adipogenesis. Proc Natl Acad Sci USA2005; 102: 9766-9771.

7. Farmer SR. Transcriptional control of adipocyte formation. Cell Metab2006; 4: 263-273.

8. Asada R, Kanemoto S, Kondo S, Saito A, Imaizumi K. The signalling from endoplasmic reticulum-resident bZIP transcription factors involved in diverse cellular physiology. J Biochem2011; 149: 507-518.

9. Fujii T, Tamura K, Masai K, Tanaka H, Nishimune Y, Nojima H. Use of stepwise subtraction to comprehensively isolate mouse genes whose transcription is up-regulated during spermiogenesis. EMBO Rep2002; 3: 367-372.

10. Nagamori I, Yomogida K, Ikawa M, Okabe M, Yabuta N, Nojima H. The testes-specific bZip type transcription factor Tisp 40 plays a role in ER stress responses and chromatin packaging during spermiogenesis. Genes Cells2006; 11: 1161-1171.

11. Nagamori I, Yabuta N, Fujii T, Tanaka H, Yomogida K, Nishimune $Y$ et al. Tisp40, a spermatid specific bZip transcription factor, functions by binding to the unfolded protein response element via the Rip pathway. Genes Cells 2005; 10: 575-594.

12. Lee MW, Chanda D, Yang J, Oh H, Kim SS, Yoon YS et al. Regulation of hepatic gluconeogenesis by an ER-bound transcription factor, CREBH. Cell Metab2010; 11: 331-339.

13. Stirling J, O'Hare P. CREB4, a transmembrane bZip transcription factor and potential new substrate for regulation and cleavage by S1P. Mol Biol Cell 2006; 17: 413-426.

14. Patel YM, Lane MD. Mitotic clonal expansion during preadipocyte differentiation: calpainmediated turnover of p27. J Biol Chem2000; 275: 17653-17660.

15. Tang QQ, Otto TC, Lane MD. Mitotic clonal expansion: a synchronous process required for adipogenesis. Proc Natl Acad Sci USA. 2003; 100: 44-49. 
16. Tong Q, Dalgin G, Xu H, Ting CN, Leiden JM, Hotamisligil GS. Function of GATA transcription factors in preadipocyte-adipocyte transition. Science 2000; 290: 134-138.

17. Wu Z, Xie Y, Bucher NL, Farmer SR. Conditional ectopic expression of C/EBP beta in NIH-3T3 cells induces PPAR gamma and stimulates adipogenesis. Genes Dev1995; 9 2350-2363.

18. Macotela Y, Emanuelli B, Mori MA, Gesta S, Schulz TJ, Tseng YH et al. Intrinsic differences in adipocyte precursor cells from different white fat depots. Diabetes 2012; 61: 1691-1699.

19. Kim JB, Spiegelman BM. ADD1/SREBP1 promotes adipocyte differentiation and gene expression linked to fatty acid metabolism. Genes Dev1996; 10: 1096-1107.

20. Kim JB, Wright HM, Wright M, Spiegelman BM. ADD1/SREBP1 activates PPARgamma through the production of endogenous ligand. Proc Natl Acad Sci USA1998; 95: 4333-4337.

21. Fajas L, Schoonjans K, Gelman L, Kim JB, Najib J, Martin G et al. Regulation of peroxisome proliferator-activated receptor gamma expression by adipocyte differentiation and determination factor $1 /$ sterol regulatory element binding protein 1 : implications for adipocyte differentiation and metabolism. Mol Cell Biol1999; 19: 5495-5503.

22. Tontonoz P, Hu E, Spiegelman BM. Stimulation of adipogenesis in fibroblasts by PPAR gamma 2, a lipid-activated transcription factor. Cell 1994; 79: 1147-1156.

23. Johnson PF. Molecular stop signs: regulation of cell-cycle arrest by C/EBP transcription factors. J Cell Sci2005; 118: 2545-2555.

24. Porse BT, Pedersen TA, Xu X, Lindberg B, Wewer UM, Friis-Hansen $L$ et al. E2F repression by C/EBPalpha is required for adipogenesis and granulopoiesis in vivo. Cell 2001; 107: 247-258.

25. Altiok S, Xu M, Spiegelman BM. PPARgamma induces cell cycle withdrawal: inhibition of E2F/DP DNA-binding activity via down-regulation of PP2A. Genes Dev1997; 11: 1987-1998.

26. Morrison RF, Farmer SR. Role of PPARgamma in regulating a cascade expression of cyclin-dependent kinase inhibitors, p18(INK4c) and p21(Waf1/Cip1), during adipogenesis. J Biol Chem1999; 274: 17088-17097.

27. Timchenko NA, Wilde M, lakova P, Albrecht JH, Darlington GJ. E2F/p107 and E2F/p130 complexes are regulated by C/EBPalpha in 3T3-L1 adipocytes. Nucleic Acids Res1999; 27 3621-3630.

28. Tong $Q$, Tsai J, Tan G, Dalgin G, Hotamisligil GS. Interaction between GATA and the C/EBP family of transcription factors is critical in GATA-mediated suppression of adipocyte differentiation. Mol Cell Biol2005; 25: 706-715.

29. Berry R, Jeffery E, Rodeheffer MS. Weighing in on adipocyte precursors. Cell Metab2014; 19: 8-20.

30. Wang QA, Tao C, Gupta RK, Scherer PE. Tracking adipogenesis during white adipose tissue development, expansion and regeneration. Nat Med 2013; 19: 1338-1344.

31. Choi H, Lee H, Kim TH, Kim HJ, Lee YJ, Lee SJ et al. G0/G1 switch gene 2 has a critical role in adipocyte differentiation. Cell Death Differ2014; 21: 1071-1080.

32. Sugii S, Kida Y, Kawamura T, Suzuki J, Vassena R, Yin YQ et al. Human and mouse adipose-derived cells support feeder-independent induction of pluripotent stem cells Proc Natl Acad Sci USA2010; 107: 3558-3563.
33. McClung JP, Roneker CA, Mu W, Lisk DJ, Langlais P, Liu F et al. Development of insulin resistance and obesity in mice overexpressing cellular glutathione peroxidase. Proc Natl Acad Sci USA2004; 101: 8852-8857.

34. Ichimura A, Hirasawa A, Poulain-Godefroy O, Bonnefond A, Hara T, Yengo $L$ et al. Dysfunction of lipid sensor GPR120 leads to obesity in both mouse and human. Nature 2012; 483: 350-354.

35. Kim TH, Kim H, Park JM, Im SS, Bae JS, Kim MY et al. Interrelationship between liver X receptor alpha, sterol regulatory element-binding protein-1c, peroxisome proliferatoractivated receptor gamma, and small heterodimer partner in the transcriptional regulation of glucokinase gene expression in liver. J Biol Chem2009; 284: 15071-15083.

36. Lee YJ, Ko EH, Kim JE, Kim E, Lee H, Choi H et al. Nuclear receptor PPARgamma-regulated monoacylglycerol 0-acyltransferase 1 (MGAT1) expression is responsible for the lipid accumulation in diet-induced hepatic steatosis. Proc Natl Acad Sci US America. 2012; 109 : 13656-13661.

37. Hwang SS, Lee S, Lee W, Lee GR. GATA-binding protein-3 regulates T helper type 2 cytokine and ifng loci through interaction with metastasis-associated protein 2. Immunology 2010; 131: 50-58.

38. Choi HK, Park SY, Oh HJ, Han EJ, Lee YH, Lee J et al. PKA negatively regulates PP2Cbeta to activate NF-kappaB-mediated inflammatory signaling. Biochem Biophys Res Commun2013; 436: 473-477.

39. Kim SJ, Choi IJ, Cheong TC, Lee SJ, Lotan R, Park SH et al. Galectin-3 increases gastric cancer cell motility by up-regulating fascin-1 expression. Gastroenterology 2010; 138: 1035-45 e1-2.

40. Grigorova B, Mara C, Hollender C, Sijacic P, Chen X, Liu Z. LEUNIG and SEUSS co-repressors regulate miR172 expression in Arabidopsis flowers. Development 2011; 138: 2451-2456.

41. Mukhopadhyay A, Deplancke B, Walhout AJ, Tissenbaum HA. Chromatin immunoprecipitation (ChIP) coupled to detection by quantitative real-time PCR to study transcription factor binding to DNA in Caenorhabditis elegans. Nat Protoc2008; 3: 698-709.

42. Tang QQ, Zhang JW, Daniel Lane M. Sequential gene promoter interactions of C/EBPbeta, C/EBPalpha, and PPARgamma during adipogenesis. Biochem Biophys Res Commun2004; 319: $235-239$

(c) Cell Death and Disease is an open-access journal published by Nature Publishing Group. This work is licensed under a Creative Commons Attribution 4.0 International Licence. The images or other third party material in this article are included in the article's Creative Commons licence, unless indicated otherwise in the credit line; if the material is not included under the Creative Commons licence, users will need to obtain permission from the licence holder to reproduce the material. To view a copy of this licence, visit http://creativecommons.org/licenses/by/4.0 